\title{
DEFAULTABLE OPTIONS IN A MARKOVIAN INTENSITY MODEL OF CREDIT RISK
}

\author{
Tomasz R. Bielecki* \\ Department of Applied Mathematics \\ Illinois Institute of Technology \\ Chicago, IL 60616, USA \\ Stéphane Crépey ${ }^{\dagger}$ \\ Département de Mathématiques \\ Université d'Évry Val d'Essonne \\ 91025 Évry Cedex, France \\ Monique Jeanblanc ${ }^{\ddagger}$ \\ Département de Mathématiques \\ Université d'Évry Val d'Essonne \\ 91025 Évry Cedex, France \\ and \\ Europlace Institute of Finance \\ Marek Rutkowski ${ }^{\S}$ \\ School of Mathematics and Statistics \\ University of New South Wales \\ Sydney, NSW 2052, Australia \\ and \\ Faculty of Mathematics and Information Science \\ Warsaw University of Technology \\ 00-661 Warszawa, Poland
}

September 16, 2008

Note to the Reader: This is an updated version of the paper forthcoming under the same title in the journal Mathematical Finance, meant for consistency with the latest developments of the companion paper [4].

\footnotetext{
*The research of T.R. Bielecki was supported by NSF Grant 0202851 and Moody's Corporation grant 5-55411.

${ }^{\dagger}$ The research of S. Crépey was supported by Ito33.

¥The research of M. Jeanblanc was supported by Ito33 and Moody's Corporation grant 5-55411.

$\S$ The research of M. Rutkowski was supported by the 2007 Faculty Research Grant PS12918.
} 


\section{Introduction}

In Bielecki et al. [4, we studied the valuation and hedging of defaultable game options in a very general reduced-form model of credit risk. Given a filtered probability space $(\Omega, \mathbb{G}, \mathbb{P})$, used to model the primary market, it was assumed in $[4$ that $\mathbb{G}=\mathbb{H} \vee \mathbb{F}$, where the filtration $\mathbb{H}$ carries the information about the default and the reference filtration $\mathbb{F}$ represents all other information available to traders. The main technique employed in 4 was the effective reduction of the information flow from the full filtration $\mathbb{G}$ to the reference filtration $\mathbb{F}$. Working under a risk-neutral probability measure $\mathbb{Q}$ and under suitable conditions on the $\mathbb{F}$ - optional projection of the default indicator process $H_{t}=\mathbb{1}_{\left\{\tau_{d} \leq t\right\}}$, we derived convenient pricing formulae with respect to the reference filtration $\mathbb{F}$. In addition, we proved that, under suitable integrability and regularity conditions embedded in the standing assumption that a related doubly reflected BSDE admits a solution under $\mathbb{Q}$, the stateprocess of this solution corresponds to the minimal (super)hedging price with a $(\mathbb{G}, \mathbb{Q}$ ) - sigma (or local, under suitable assumptions) martingal ${ }^{1}$ cost. This result is actually interesting even beyond the scope of credit risk, as it provides a general connection between, on the one hand, arbitrage prices of an option (a game option, including American and European options as special cases), defaultable or not (the latter case corresponding to $\tau_{d}=\infty$ ), and, on the other hand, a suitable notion of hedging with a sigma (or local) martingale cost, in a general, possibly incomplete, market. In the special case of a complete market, the cost of the related hedging strategies vanishes, and the hedging strategies are super-hedges in the usual sense.

For an efficient practical implementation, a (dynamic) pricing model should possess a suitable Markovian property. For this reason, we propose in this paper a generic Markovian pre-default intensity model of credit risk, which encompasses as a special case the jump-diffusion model studied in detail in [5] (cf. Subsection 4.3 of the present paper). As a prerequisite, we recall in Theorem 2.1 (a variant of) the main results from [4. As compared with [4, we work in this paper under the slightly stronger assumption that the doubly reflected $\operatorname{BSDE}(\mathcal{E})$ associated with a defaultable game option has a solution $(\Theta, M, K)$ where $K$ is a continuous process and $M$ belongs to $\mathcal{H}^{2}$. Though unnecessary from the strictly mathematical point of view (see 4]), the latter requirements are important in view of practical use of our previous results, like showing that $(\mathcal{E})$ is well-posed, establishing the connection with a PDE formulation of the problem in Markovian settings, devising appropriate numerical approximation schemes, etc.

It should be made clear that in our previous work [4, we simply postulated that a primary market arbitrage price process $X$ is given and it satisfies all our assumptions. We did not address the issues of proving existence and/or building such market models. In order to fill this gap, we develop in Subsection 3.1 a generic method of constructing such an arbitrage price process $X$ (see Proposition 3.1). In particular, we provide in Lemma 3.1 (see also Corollary 3.1) a general condition which should be imposed on a pre-default primary market model in order to make the model arbitrage-free.

Under a rather generic specification of the infinitesimal generator of a driving Markov factor process, we subsequently develop in Subsections 3.2 to 3.4 the variational inequality approach (cf. (34)) to pricing and hedging of a defaultable game option. Let us stress again that putting the previous theoretical results in a Markovian framework is a necessary step towards any implementation. The generic Markovian model considered in this paper is also interesting as a concrete example of the otherwise abstract material presented in 4] or in Section 2 (see Theorems 2.1] and 2.2) of this paper.

Finally, in Section 4, we illustrate our study by considering convertible bonds. We specify to this case the general variational inequality (34) and we emphasize the crucial role of the freedom to choose the most convenient driver (i.e., the parameter process $F$ ) in equation $(\mathcal{E})$.

\footnotetext{
${ }^{1}$ Sigma martingales are a relevant generalization of local martingales, see, for instance, $7,18,24$.
} 


\subsection{General Set-Up}

For a finite horizon date $T>0$, we assume that the primary market is composed of the saving account and of $d$ risky assets with price processes defined on a filtered probability space $(\Omega, \mathbb{G}, \mathbb{P})$, where $\mathbb{P}$ denotes the statistical probability measure. We postulate that (cf. $\underline{3}$ ):

- the discount factor process $\beta$, that is, the inverse of the savings account, is a $\mathbb{G}$-adapted, finite variation, continuous, positive and bounded process;

- the prices of risky assets are $\mathbb{G}$-semimartingales with càdlàg sample paths.

The primary risky assets, with $\mathbb{R}^{d}$-valued price process $X$, are assumed to pay dividends, whose cumulative value process, denoted by $\mathcal{D}$, is modeled as a $\mathbb{G}$-adapted, càdlàg and $\mathbb{R}^{d}$-valued process of finite variation. Given the price process $X$, we define the cumulative price $\widehat{X}$ of the asset as

$$
\widehat{X}_{t}=X_{t}+\beta_{t}^{-1} \int_{[0, t]} \beta_{u} d \mathcal{D}_{u}
$$

We assume that $\beta \widehat{X}$ is locally bounded and that the primary market model is free of arbitrage opportunities (though presumably incomplete), in the sense that there exists a risk-neutral measure $\mathbb{Q} \in \mathcal{M}$, where $\mathcal{M}$ denotes the set of probability measures $\mathbb{Q}$ equivalent to $\mathbb{P}$ for which $\beta \widehat{X}$ is a $(\mathbb{G}, \mathbb{Q})$ - local martingale.

Note that relaxing the assumption of local boundness on $\beta \widehat{X}$, the only statement that would change in this paper is the previous one, namely the characterization of arbitrages prices. This characterization would then be in terms of $(\mathbb{G}, \mathbb{Q})$ - sigma martingales rather than in terms of $(\mathbb{G}, \mathbb{Q})$ - local martingales. Since we want to avoid the notion of sigma martingales in this paper, to keep it more user's friendly, we prefer to work under this harmless assumption on $\beta \widehat{X}$. We refer the interested reader to [4 for the most general results under minimal assumptions.

In this paper, similarly as in [3, 4, we work with the notion of a vector (as opposed to componentwise) stochastic integral (see Cherny and Shiryaev [7]). By convention, we denote by $\int_{0}^{t}$ the integral over $(0, t]$; otherwise, we explicitly specify the domain of integration as a subscript of $\int$. Also note that in what follows we in fact deal with right-continuous and completed versions of all relevant filtrations, so that all the filtrations under consideration satisfy the so-called 'usual conditions.'

\section{Valuation and Hedging of Defaultable Options in the Haz- ard Process Set-Up: A User's Guide}

In [4, we derived general hedging results for a game option under fairly general assumptions in the so-called hazard process set-up. In the same framework, and at the cost of slightly stronger assumptions (see Remark A.1(i) in the Appendix), we shall now derive variants of these results that are required in practical applications of the general theory.

In this section, we work under a risk-neutral measure $\mathbb{Q}$ which is fixed throughout. So all the measure-dependent notions like (local) martingale, compensator, etc., implicitly refer to the probability measure $\mathbb{Q}$.

\subsection{Hazard Process Set-Up}

Given a $[0,+\infty]$-valued $\mathbb{G}$ - stopping time $\tau_{d}$ representing the default time of a reference entity, we assume that $\mathbb{G}=\mathbb{H} \vee \mathbb{F}$, where the filtration $\mathbb{H}$ is generated by the default indicator process $H_{t}=\mathbb{1}_{\left\{\tau_{d} \leq t\right\}}$ and $\mathbb{F}$ is some reference filtration. We assume that the process $G$ given by $G_{t}=$ $\mathbb{Q}\left(\tau_{d}>t \mid \mathcal{F}_{t}\right)$ for $t \in \mathbb{R}_{+}$is (strictly) positive, continuous and non-increasing. Hence the $\mathbb{F}$ - hazard process $\Gamma_{t}=-\ln \left(G_{t}\right)$ of $\tau_{d}$ is well defined, continuous and non-decreasing on $\mathbb{R}^{+}$. The $\mathbb{G}$ - stopping 
time $\tau_{d}$ is then an $\mathbb{F}$ - pseudo-stopping time ([23], see also [4]), which means in particular that any $\mathbb{F}$ - local martingale stopped at $\tau_{d}$ is a $\mathbb{G}$ - local martingale (cf. [23, Theorem 4]).

It is also postulated throughout Section 2 that the default time $\tau_{d}$ avoids $\mathbb{F}$ - stopping times, that is, $\mathbb{Q}\left(\tau_{d}=\tau\right)=0$ for any $\mathbb{F}$ - stopping time $\tau$. Under the continuity assumption on $\Gamma$, this would for instance (but not only) be the case under the hypothesis (not made in this paper) that any $\mathbb{F}$-martingale is continuous, see Mansuy and Yor [22, p.25]. The standing assumption that $\tau_{d}$ avoids $\mathbb{F}$ - stopping times implies, in particular, that an càdlàg process $Y$ cannot jump at $\tau_{d}$, that is, $\Delta Y_{\tau_{d}}:=Y_{\tau_{d}}-Y_{\tau_{d}-}=0$, almost surely.

We shall sometimes assume, in addition, that the $\mathbb{F}$-adapted processes $\beta$ and $\Gamma$ are absolutely continuous with respect to the Lebesgue measure, specifically:

- $\beta_{t}=\exp \left(-\int_{0}^{t} r_{u} d u\right)$ for an $\mathbb{F}$-adapted, bounded from below short-term interest rate process $r$, - $\Gamma_{t}=\int_{0}^{t} \gamma_{u} d u$, for a non-negative $\mathbb{F}$-adapted process $\gamma$, called the $\mathbb{F}$ - intensity process of $\tau_{d}$.

A set-up satisfying the latter assumptions will be referred to as a default intensity set-up.

We now recall the concept of a (dividend paying) defaultable game option (see [20, 19, 3, 4]) with inception date 0 and maturity date $T$.

For any $t \in[0, T]$, let $\mathcal{F}_{T}^{t}$ (resp. $\mathcal{G}_{T}^{t}$ ) denote the set of $[t, T]$-valued $\mathbb{F}$ (resp. $\mathbb{G}$ )-stopping times; given a further $\bar{\tau} \in \mathcal{F}_{T}^{0}$, let $\overline{\mathcal{G}}_{T}^{t}$ stand for $\left\{\tau \in \mathcal{G}_{T}^{t} ; \tau \wedge \tau_{d} \geq \bar{\tau} \wedge \tau_{d}\right\}$.

The stopping time $\bar{\tau} \in \mathcal{F}_{T}^{0}$ in the following definition is used to model the restriction that the issuer of a game option may be prevented from calling the option during some random time interval $[0, \bar{\tau})$ (see [3] ). Let $\overline{\mathcal{G}}_{T}^{t}$ stand for $\left\{\tau \in \mathcal{G}_{T}^{t} ; \tau \wedge \tau_{d} \geq \bar{\tau} \wedge \tau_{d}\right\}$.

Definition 2.1 A defaultable game option with lifting time of the call protection $\bar{\tau} \in \mathcal{F}_{T}^{0}$, is a game option with the ex-dividend cumulative discounted cash flows $\beta_{t} \pi\left(t ; \tau_{p}, \tau_{c}\right)$ given by the formula, for any $t \in[0, T]$ and $\left(\tau_{p}, \tau_{c}\right) \in \mathcal{G}_{T}^{t} \times \overline{\mathcal{G}}_{T}^{t}$,

$$
\beta_{t} \pi\left(t ; \tau_{p}, \tau_{c}\right)=\int_{t}^{\tau} \beta_{u} d D_{u}+\mathbb{1}_{\left\{\tau_{d}>\tau\right\}} \beta_{\tau}\left(\mathbb{1}_{\left\{\tau=\tau_{p}<T\right\}} L_{\tau_{p}}+\mathbb{1}_{\left\{\tau<\tau_{p}\right\}} U_{\tau_{c}}+\mathbb{1}_{\{\tau=T\}} \xi\right),
$$

where $\tau=\tau_{p} \wedge \tau_{c}$ and where

- the dividend process $D=\left(D_{t}\right)_{t \in[0, T]}$ equals

$$
D_{t}=\int_{[0, t]}\left(1-H_{u}\right) d C_{u}+R_{u} d H_{u}
$$

for some coupon process $C=\left(C_{t}\right)_{t \in[0, T]}$, which is a $\mathbb{F}$-predictable càdlàg process with bounded variation, and some real-valued, $\mathbb{F}$-predictable, locally bounded recovery process $R=\left(R_{t}\right)_{t \in[0, T]}$,

- the put payment $L=\left(L_{t}\right)_{t \in[0, T]}$ and the call payment $U=\left(U_{t}\right)_{t \in[0, T]}$ are $\mathbb{F}$-adapted, real-valued, càdlàg processes,

- the inequality $L_{t} \leq U_{t}$ holds for every $t \in\left[\tau_{d} \wedge \bar{\tau}, \tau_{d} \wedge T\right)$, and

- the payment at maturity $\xi$ is a real-valued, $\mathcal{F}_{T}$-measurable random variable.

Note that $\pi\left(t ; \tau_{p}, \tau_{c}\right)$ is a $\mathcal{G}_{\tau \wedge \tau_{d}}$-measurable random variable.

We further assume that the cumulative discounted payoff is bounded from below. Specifically, there exists a constant $c$ such that

$$
\beta_{t} \widehat{\mathcal{L}}_{t}:=\int_{[0, t]} \beta_{u} d D_{u}+\mathbb{1}_{\left\{\tau_{d}>t\right\}} \beta_{t}\left(\mathbb{1}_{\{t<T\}} L_{t}+\mathbb{1}_{\{t=T\}} \xi\right) \geq-c, \quad t \in[0, T] .
$$

In order to get an upper bound for this payoff, we shall sometimes assume that there exists a constant $c$ such that

$$
\beta_{t} \widehat{\mathcal{U}}_{t}:=\int_{[0, t]} \beta_{u} d D_{u}+\mathbb{1}_{\left\{\tau_{d}>t\right\}} \beta_{t}\left(\mathbb{1}_{\{t<T\}} U_{t}+\mathbb{1}_{\{t=T\}} \xi\right) \leq c, \quad t \in[0, T] .
$$


The class of defaultable game options covers as special cases defaultable American options (case $\bar{\tau}=T$ ). It can be shown that the latter class includes defaultable European options as a special case (sub-case where the maximum of $\beta \widehat{\mathcal{L}}$ is attained at $T$, see [3]).

One defines likewise defaultable European options as contracts with cash flows $\phi(t)$ given by, for $t \in[0, T]$,

$$
\beta_{t} \phi(t)=\int_{t}^{T} \beta_{u} d D_{u}+\mathbb{1}_{\left\{\tau_{d}>T\right\}} \beta_{T} \xi
$$

Defaultable European options can equivalently be redefined as the sub-case of defaultable American options for which the maximum of $\beta \widehat{\mathcal{L}}$ is attained at $T$ (see [3]).

All options considered in this paper are potentially defaultable, so the expressions option and defaultable option will be used interchangeably in the sequel.

We are now in the position to introduce the concept of hedging of a game option. Recall that $X$ (resp. $\widehat{X}$ ) is the price process (resp. cumulative price process) of primary traded assets, cf. (1).

Definition 2.2 By a (self-financing) primary trading strategy we mean a pair $\left(V_{0}, \zeta\right)$ such that:

- $V_{0}$ is a $\mathcal{G}_{0}$-measurable real-valued random variable representing the initial wealth,

- $\zeta$ is an $\mathbb{R}^{1 \otimes d}$-valued, $\beta \widehat{X}$-integrable process representing holdings in primary risky assets.

The wealth process $V$ of $\left(V_{0}, \zeta\right)$ satisfies, for $t \in[0, T]$,

$$
d\left(\beta_{t} V_{t}\right)=\zeta_{t} d\left(\beta_{t} \widehat{X}_{t}\right)
$$

with an initial condition $V_{0}$.

Definition 2.3 (i) An issuer hedge with cost process $\rho$ for the game option with ex-dividend cumulative discounted cash flows $\beta \pi$ (cf. (2)) is represented by a quadruplet $\left(V_{0}, \zeta, \rho, \tau_{c}\right)$ such that:

- $\left(V_{0}, \zeta\right)$ is a primary strategy with the wealth process $V$ given by (5),

- a cost process $\rho$ is a real-valued $\mathbb{G}$-semimartingale with $\rho_{0}=0$,

- a (fixed) call time $\tau_{c}$ belongs to $\overline{\mathcal{G}}_{T}^{0}$,

- the following inequality is valid, for every put time $\tau_{p} \in \mathcal{G}_{T}^{0}$,

$$
\beta_{\tau} V_{\tau}+\int_{0}^{\tau} \beta_{u} d \rho_{u} \geq \beta_{0} \pi\left(0 ; \tau_{p}, \tau_{c}\right), \quad \text { a.s. }
$$

(ii) A holder hedge with cost process $\rho$ for the game option is a quadruplet $\left(V_{0}, \zeta, \rho, \tau_{p}\right)$ such that:

- $\left(V_{0}, \zeta\right)$ is a primary strategy with the wealth process $V$ given by (5),

- a cost process $\rho$ is a real-valued $\mathbb{G}$-semimartingale with $\rho_{0}=0$,

- a (fixed) put time $\tau_{p}$ belongs to $\mathcal{G}_{T}^{0}$,

- the following inequality is valid, for every call time $\tau_{c} \in \overline{\mathcal{G}}_{T}^{0}$,

$$
\beta_{\tau} V_{\tau}+\int_{0}^{\tau} \beta_{u} d \rho_{u} \geq-\beta_{0} \pi\left(0 ; \tau_{p}, \tau_{c}\right), \quad \text { a.s. }
$$

Issuer or holder hedges at no cost (that is, with $\rho=0$ ) are thus in effect issuer or holder superhedges.

Remarks 2.1 (i) The process $\rho$ can also be interpreted as the (running) financing cost, that is, the amount of cash added to (if $d \rho_{t} \geq 0$ ) or withdrawn from (if $d \rho_{t} \leq 0$ ) the hedging portfolio in order to get a (not self-financing any longer) superhedge.

(ii) Regarding the admissibility issues (see, e.g., Delbaen and Schachermayer [12]), note that the l.h.s. of (6) (discounted wealth process with financing costs included) is bounded from below for any issuer hedge $\left(V_{0}, \zeta, \rho, \tau_{c}\right)$. Likewise, in the case of a bounded payoff $\pi$ (that is, assuming (3)), the l.h.s. of 77) (discounted wealth process with financing costs included) is bounded from below for any holder hedge $\left(V_{0}, \zeta, \rho, \tau_{p}\right)$. 
Let us now consider the special case of a defaultable European option with cash-flows $\phi$ (cf. (4)).

Definition 2.4 (i) An issuer hedge with cost $\rho$ (a real-valued $\mathbb{G}$-semimartingale with $\rho_{0}=0$ ) for a defaultable European option is a primary strategy $\left(V_{0}, \zeta\right)$ with wealth process $V$ such that

$$
\beta_{T} V_{T}+\int_{0}^{T} \beta_{u} d \rho_{u} \geq \beta_{0} \phi(0), \quad \text { a.s. }
$$

If the inequality may be replaced by equality then we deal with an issuer replicating strategy with cost $\rho$.

(ii) A holder hedge with cost $\rho$ (a real-valued $\mathbb{G}$-semimartingale with $\rho_{0}=0$ ) for a defaultable European option is a primary strategy $\left(V_{0}, \zeta\right)$ with wealth process $V$ such that

$$
\beta_{T} V_{T}+\int_{0}^{T} \beta_{u} d \rho_{u} \geq-\beta_{0} \phi(0), \quad \text { a.s. }
$$

If the inequality may be replaced by equality then we deal with a holder replicating strategy with cost $\rho$.

\subsection{Valuation and Hedging Results}

We will now study valuation and hedging of a game option under suitable integrability and regularity conditions. These conditions are implicitly embedded in the standing assumption that a related doubly reflected $\operatorname{BSDE}(\mathcal{E})$, stated under the risk-neutral measure $\mathbb{Q}$, admits a solution. Assuming that $(\mathcal{E})$ has a solution (which will hold under mild conditions, cf. the discussion following Definition 2.5 , we shall deduce explicit hedging strategies with minimal initial wealth and a $(\mathbb{G}, \mathbb{Q})$ - local martingale cost for a game option.

Let $\alpha_{t}=\beta_{t} \exp \left(-\Gamma_{t}\right)$ denote the credit-risk adjusted discount factor; note that the process $\alpha$ is bounded. We define the $\mathbb{F}$-adapted processes $\bar{D}$ and $\bar{F}$ of finite variation by setting, for $t \in[0, T]$,

$$
\bar{D}_{t}=\int_{[0, t]}\left(d C_{u}+R_{u} d \Gamma_{u}\right), \bar{F}_{t}:=\alpha_{t}^{-1} \int_{[0, t]} \alpha_{u} d \bar{D}_{u}
$$

Note that from the point of view of the financial interpretation, the process $\bar{D}$ represents the dividend process (including the recovery payment) as seen in an equivalent fictitious default-free world (see [4).

Let $F$ be a given $\mathbb{F}$-adapted, finite variation process, such that $\bar{F}-F$ be bounded from below; we shall refer to the process $F$ as the driver in what follows. We introduce the r.v. $\chi$ and the processes $\mathcal{L}, \overline{\mathcal{U}}$ defined as

$$
\chi=\xi+\bar{F}_{T}-F_{T}, \quad \mathcal{L}=L+\bar{F}-F, \quad \overline{\mathcal{U}}=\mathbb{1}_{\{t<\bar{\tau}\}} \infty+\mathbb{1}_{\{t \geq \bar{\tau}\}} \mathcal{U}
$$

with $\mathcal{U}=U+\bar{F}-F$.

We consider the following doubly reflected $B S D E(\mathcal{E})$ with data $F, \chi, \mathcal{L}, \mathcal{U}, \bar{\tau}$ (see Definition 2.5):

$$
\left.\begin{array}{c}
\Theta_{t}=\chi+\left(F_{T}-F_{t}\right)-\int_{t}^{T}\left(F_{u}+\Theta_{u}\right) d B_{u}+K_{T}-K_{t}-\left(M_{T}-M_{t}\right), \quad t \in[0, T], \\
\mathcal{L}_{t} \leq \Theta_{t} \leq \overline{\mathcal{U}}_{t}, \quad t \in[0, T], \\
\int_{0}^{T}\left(\Theta_{u}-\mathcal{L}_{u}\right) d K_{u}^{+}=\int_{0}^{T}\left(\overline{\mathcal{U}}_{u}-\Theta_{u}\right) d K_{u}^{-}=0,
\end{array}\right\}(\mathcal{E})
$$

where $B=-\ln \alpha$. Note that since $d\left(\alpha_{t} F_{t}\right)=\alpha_{t}\left(d F_{t}-F_{t} d B_{t}\right)$, we have that

$$
F_{T}-F_{t}-\int_{t}^{T} F_{u} d B_{u}=\int_{t}^{T} \alpha_{u}^{-1} d\left(\alpha_{u} F_{u}\right)
$$


and likewise for $\Theta$. Hence the first line in $(\mathcal{E})$ can be rewritten as

$$
\Theta_{t}=\chi-\int_{t}^{T} \Theta_{u} d B_{u}+\int_{t}^{T} \alpha_{u}^{-1} d\left(\alpha_{u} F_{u}\right)+K_{T}-K_{t}-\left(M_{T}-M_{t}\right), \quad t \in[0, T],
$$

Finally, let us consider the special case of a default intensity set-up, so $B_{t}=-\ln \alpha_{t}=\int_{0}^{t} \mu_{u} d u$ with $\mu=r+\gamma$, and of a driver $F$ of the form

$$
F_{t}=\alpha_{t}^{-1} \int_{0}^{t} \alpha_{u} f_{u} d u
$$

for some $\mathbb{F}$-adapted time-integrable process $f$ over $[0, T]$ such that the process $\bar{F}-F$ is bounded from below. Then $(9)$ can be rewritten as

$$
\Theta_{t}=\chi+\int_{t}^{T}\left(f_{u}-\mu_{u} \Theta_{u}\right) d u+K_{T}-K_{t}-\left(M_{T}-M_{t}\right), \quad t \in[0, T] .
$$

In this case the related driver terms in $(\mathcal{E})$ are thus given as integrals with respect to the Lebesgue measure, which is the standard form in the BSDE literature (see e.g. [10, 16, 11, 14]).

Let $\mathcal{H}^{2}$ denote the set of $\mathbb{F}$-martingales with integrable quadratic variation over $[0, T]$ vanishing at time 0 .

Definition 2.5 By a solution to $(\mathcal{E})$, we mean a triplet $(\Theta, M, K)$ such that:

- the state process $\Theta$ is a real-valued, $\mathbb{F}$-adapted, càdlàg process,

- $M$ lies in $\mathcal{H}^{2}$,

- $K$ is an $\mathbb{F}$-adapted finite variation continuous process vanishing at time 0 ,

- all conditions in $(\mathcal{E})$ are satisfied, where in the third line $K^{+}$and $K^{-}$denote the Jordan components of $K$ (i.e., the decomposition $K=K^{+}-K^{-}$where the non-decreasing continuous processes $K^{ \pm}$ vanish at time 0 and define mutually singular measures), and where the convention that $0 \times \pm \infty=0$ is made in the third line.

For various specifications of the present set-up and sets of technical assumptions ensuring the existence and uniqueness of a solution to $(\mathcal{E})$, we refer the reader to [10, 16, 15, 11. Basically, for the data in suitable spaces of square-integrable processes and random variables, and allowing for jumps of $\mathcal{L}$ and $\mathcal{U}$ (at totally inaccessible $\mathbb{F}$ - stopping times, see Subsection 4.4, the existence of a solution to $(\mathcal{E})$ (supplemented by suitable integrability conditions on the data and the solution) is essentially equivalent to the so-called Mokobodski condition, namely, the existence of a quasimartingale $Y$ such that $\mathcal{L} \leq Y \leq \mathcal{U}$ on $[0, T]$ (see Crépey and Matoussi [10, Hamadène and Hassani [16, Theorem 4.1], and previous works in this direction, starting with [11]).

Recall that a quasimartingale $Y$ can equivalently be defined as a difference of two non-negative supermartingales, or in terms of a bound on some conditionally expected variations of $Y$ on arbitrary partitions of $[0, T]$, or as a special semimartingale with predictable finite variation component of integrable variation (Protter [24, Chapter III, section 4]).

In particular, any square integrable Itô-Lévy process $S$ (Itô-Lévy process with square integrable special semimartingale decomposition components), or $S \vee \ell$ for any such process $S$ and constant $\ell$, is a quasimartingale (see Crépey and Matoussi [10]). Hence the Mokobodski condition is satisfied, and the existence of a solution for $(\mathcal{E})$ holds, whenever $\mathcal{L}$ and/or $\mathcal{U}$ is given by $S$ or $S \vee \ell$ for such an Itô-Lévy process $S$, as it is the case in many practical applications (see [10, 5]).

Note that equation $(\mathcal{E})$ (including the definition of the barriers $\mathcal{L}$ and $\mathcal{U}$ in $(\mathcal{E})$ ) and its consequences are implicitly parameterized by the choice of a driver $F$ in $(\mathcal{E})$. In fact $(\Theta, M, K)$ solves equation $(\mathcal{E})$ for some driver $F$ if and only if $(\widehat{\Theta}, M, K)$, where $\widehat{\Theta}=\Theta+F$, solves equation $(\mathcal{E})$ for $F=0$ (and accordingly modified barriers). Yet, as we shall see in a concrete example in Section 4 (see, in particular, Subsection 4.4), the freedom of choosing the most convenient driver is important in financial applications. So a particular form of $F$ may be selected in order to deal with the most 
tractable BSDE, namely, the BSDE with the simplest form of reflecting barriers, which are the most difficult point to tackle with, from the point of view of solving the BSDE (see Section 4 and $[10,5]$ ). In the case of Markovian models (see later sections and [8, [5]), this freedom will allow us to deal with the related variational inequalities of the most tractable structure.

Given a solution $(\Theta, M, K)$ to $(\mathcal{E})$, we define the $\mathbb{Q}$ - pre-default price, price, cumulative value, and optimal stopping times, respectively, by, for $t \in[0, T]$,

$$
\begin{gathered}
\widetilde{\Pi}_{t}=\Theta_{t}+F_{t}-\bar{F}_{t}, \quad \Pi_{t}=\mathbb{1}_{\left\{t<\tau_{d}\right\}} \widetilde{\Pi}_{t}, \quad \widehat{\Pi}_{t}=\Pi_{t}+\beta_{t}^{-1} \int_{[0, t]} \beta_{u} d D_{u} \\
\tau_{p}^{*}(t)=\inf \left\{u \in[t, T] ; \widetilde{\Pi}_{u} \leq L_{u}\right\} \wedge T, \quad \tau_{c}^{*}(t)=\inf \left\{u \in[\bar{\tau} \vee t, T] ; \widetilde{\Pi}_{u} \geq U_{u}\right\} \wedge T .
\end{gathered}
$$

Let $N^{d}=H-\Gamma \cdot \wedge \tau_{d}$ stand for the compensated jump-to-default process. Recall that the process $N^{d}$ is a $\mathbb{G}$-martingale, under our standing assumption that the $\mathbb{F}$ - hazard process $\Gamma$ of $\tau_{d}$ is nondecreasing and continuous.

The following statement follows by application of the main results of [4] (see the Appendix). The notion of an arbitrage price of a game option referred to in point (i) below is a suitable extension to game options (Definition 2.6 in Kallsen and Kühn [19, see also [3]) of the No Free Lunch with Vanishing Risk (NFLVR) condition of Delbaen and Schachermayer [12, 7].

Theorem 2.1 Assume that the BSDE (E) has a solution $(\Theta, M, K)$. Then :

(i) $\Pi$ is an arbitrage price process for the game option;

(ii) The process $m$ given by the formula, for $t \in[0, T]$,

$$
m_{t}=\beta_{t} \widehat{\Pi}_{t}+\int_{\left[0, t \wedge \tau_{d}\right]} \beta_{u} d K_{u}
$$

is a $\mathbb{G}$-martingale (stopped at $\tau_{d}$ ), such that

$$
d m_{t}=\mathbb{1}_{\left\{t \leq \tau_{d}\right\}} \beta_{t}\left(d M_{t}+\left(R_{t}-\widetilde{\Pi}_{t-}\right) d N_{t}^{d}\right) ;
$$

(iii) Given an arbitrary $\mathbb{R}^{1 \otimes d}$-valued, predictable and locally bounded process $\zeta$, let the process $\rho=$ $\rho(\zeta)$ be defined by $\rho_{0}=0$ and, for $t \in[0, T]$,

$$
\beta_{t} d \rho_{t}=d m_{t}-\zeta_{t} d\left(\beta_{t} \widehat{X}_{t}\right) .
$$

Then $\left(\Pi_{0}, \zeta, \rho(\zeta), \tau_{c}^{*}(0)\right)$ is an issuer hedge with $\mathbb{G}$ - local martingale cost, and $\left(-\Pi_{0},-\zeta,-\rho(\zeta), \tau_{p}^{*}(0)\right)$ (cf. (13)) is a holder hedge with $\mathbb{G}$ - local martingale cost.

(iv) $\Pi_{0}$ is the minimal initial wealth of an issuer hedge with $\mathbb{G}$ - local martingale cost and, under condition (3), $-\Pi_{0}$ is the minimal initial wealth of a holder hedge with $\mathbb{G}$ - local martingale cost.

Remarks 2.2 (i) In view of 14 the process $m$ can equivalently be redefined as the $\mathbb{G}$-local martingale component of the discounted cumulative $\mathbb{Q}$-value process $\beta \widehat{\Pi}$. The processes $m$ and $\beta \widehat{\Pi}$ are easily seen to coincide on the random interval $\left[0, \tau_{c}^{*}(0) \wedge \tau_{p}^{*}(0) \wedge \tau_{d} \wedge T\right]$ and thus both can be interpreted on this interval as the discounted cumulative $\mathbb{Q}$-value of a defaultable game option.

(ii) One defines the hedging error process (also known as the tracking error or the profit and loss process) $e=e(\zeta)$ relative to the ex-dividend $\mathbb{Q}$-price process $\Pi$, as seen from the perspective of an option's issuer, by, for $t \in[0, T]$ :

$$
\beta_{t} e_{t}=\beta_{0} \widehat{\Pi}_{0}+\int_{0}^{t} \zeta_{u} d\left(\beta_{u} \widehat{X}_{u}\right)-\beta_{t} \widehat{\Pi}_{t} .
$$

Using (14) there comes, for $t \in[0, T]$,

$$
\beta_{t} e_{t}=\int_{0}^{t \wedge \tau_{d}} \beta_{u} d K_{u}-\int_{0}^{t} \beta_{u} d \rho_{u} .
$$

Therefore $\int_{0}^{*} \beta_{t} d \rho_{t}$ can also be interpreted as the $\mathbb{G}$-local martingale component of the $\mathbb{G}$-special semimartingale $\beta e$. 
In the case of a European option, we consider the $\operatorname{BSDE}(\mathcal{E})$ with $\mathcal{L}$ replaced by $\overline{\mathcal{L}}$ such that $\alpha \overline{\mathcal{L}}=-(c+1)$, where $-c$ is a lower bound on $\alpha_{T} \chi$. Note that under mild technical assumptions this equation has a solution $(\Theta, m, K=0)$ (see [4, 10]), so that $(\mathcal{E})$ effectively reduces to a standard BSDE with no process $K$ involved.

We then define the $\mathbb{Q}$ - pre-default price, price and cumulative value as, respectively

$$
\widetilde{\Phi}_{t}=\Theta_{t}+F_{t}-\bar{F}_{t}, \quad \Phi_{t}=\mathbb{1}_{\left\{t<\tau_{d}\right\}} \widetilde{\Phi}_{t}, \quad \widehat{\Phi}_{t}=\Phi_{t}+\beta_{t}^{-1} \int_{[0, t]} \beta_{u} d D_{u}
$$

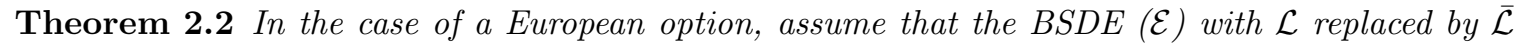
and with $\bar{\tau}=T$ has a solution $(\Theta, M, K=0)$. Then:

(i) $\Phi$ is an arbitrage price process for the option;

(ii) The discounted cumulative $\mathbb{Q}$-value process $m=\beta \widehat{\Phi}$ is a $\mathbb{G}$-martingale (stopped at $\tau_{d}$ ), such that

$$
d m_{t}=\mathbb{1}_{\left\{t \leq \tau_{d}\right\}} \beta_{t}\left(d M_{t}+\left(R_{t}-\widetilde{\Phi}_{t-}\right) d N_{t}^{d}\right)
$$

(iii) Given an arbitrary $\mathbb{R}^{1 \otimes d}$-valued, predictable and locally bounded process $\zeta$, let the process $\rho=$ $\rho(\zeta)$ be defined by by $\rho_{0}=0$ and (16) with $m \beta \widehat{\Phi}$ therein. Then $\left(\Phi_{0}, \zeta, \rho(\zeta)\right)$ is an issuer replicating strategy with $\mathbb{G}$ - local martingale cost, and $\left(-\Phi_{0},-\zeta,-\rho(\zeta)\right)$ is a holder replicating strategy with $\mathbb{G}$ - local martingale cost.

(iv) $\Phi_{0}$ is the minimal initial wealth of an issuer replicating strategy (or hedge) with $\mathbb{G}$ - local martingale cost and, for bounded $R$ and $\xi,-\Phi_{0}$ is the minimal initial wealth of a holder replicating strategy (or hedge) with $\mathbb{G}$ - local martingale cost.

So, in the European case, the process $m$ exactly corresponds to the discounted cumulative $\mathbb{Q}$-value process of the option, and $-\int_{0}^{\cdot} \beta_{t} d \rho_{t}=\beta e=\beta_{0} \widehat{\Phi}_{0}+\int_{0}^{\cdot} \zeta_{t} d\left(\beta_{t} \widehat{X}_{t}\right)-\beta \widehat{\Phi}$.

Remarks 2.3 In Theorem 2.1 or 2.2

(i) The special case $\rho=0$ corresponds to a particular form of a model completeness (attainability of defaultable European claims, cf. Theorem 2.2, see also [5]) in which the issuer (or the holder) of the option is able and wishes to hedge all risks embedded in the option. The case where $\rho \neq 0$ corresponds to either model incompleteness or the situation of a complete model in which the issuer (or the holder) is able to hedge, but she prefers not to hedge all the risks embedded in the option; for instance, she may be willing to take some bets in specific risk directions.

(ii) In cases where $\rho$ may be taken equal to 0 , the minimality statements in parts (i) of these theorems may be used to prove uniqueness of the related arbitrage prices (see [4, [5]).

\section{Markovian Set-Up}

\subsection{Market Model Factory}

In the previous sections we took a primary market model satisfying all assumptions as given. The goal of this section is to present a generic construction of an arbitrage-free primary market model in a default intensity set-up.

To this end, we assume that we are given a stochastic basis $(\Omega, \mathbb{F}, \mathbb{Q})$ endowed with the following processes:

- an $\mathbb{F}$-adapted, bounded from below and time-integrable process $r$, which is intended to represent short-term interest rate,

- an $\mathbb{F}$-adapted, non-negative and time-integrable process $\gamma$, which represents the default intensity,

- an $\mathbb{R}^{d}$-valued càdlàg $\mathbb{F}$-semimartingale $\widetilde{X}$, which is aimed to model the pre-default prices of primary assets, as well as the associated coupon process $\mathcal{C}$ and recovery process $\mathcal{R}$, such that: 
- $\mathcal{C}$ is an $\mathbb{R}^{d}$-valued, $\mathbb{F}$-predictable process of integrable variation, ${ }^{2}$

- $\mathcal{R}$ is an $\mathbb{R}^{d}$-valued, $\mathbb{F}$-predictable and bounded from below process.

Relevant ways to construct such primary data $(\Omega, \mathbb{F}, \mathbb{Q}), r, \gamma, \widetilde{X}, \mathcal{C}, \mathcal{R}$ will be given later in this section (see Remark 3.1(i)). In this subsection, all the measure-dependent notions implicitly refer to the probability measure $\mathbb{Q}$.

Given these primary data, the construction of the primary market model goes as follows. First, we define the discount factor $\beta_{t}=e^{-\int_{0}^{t} r_{u} d u}$. Next, the so-called canonical construction yields a convenient method of defining a random time $\tau_{d}$ on an enlarged probability space $(\Omega, \mathcal{G}, \mathbb{Q})$, such that (see, e.g., [6]):

- $\tau_{d}$ is a $\mathbb{G}$ - stopping time with respect to $\mathbb{G}=\mathbb{H} \vee \mathbb{F}$, where $\mathbb{H}$ is the filtration generated by the default indicator process $H_{t}=\mathbb{1}_{\left\{\tau_{d} \leq t\right\}}$,

- the process $\gamma$ is the $\mathbb{F}$ - intensity process of $\tau_{d}$, with related $\mathbb{F}$ - hazard process $\Gamma=\int_{0}^{\cdot} \gamma_{t} d t$, and thus $\tau_{d}$ is an $\mathbb{F}$ - pseudo-stopping time (cf. Section 2.1),

- $\tau_{d}$ avoids $\mathbb{F}$ - stopping times (under the canonical construction, this property can be shown by conditioning with respect to $\mathcal{F}_{T}$ ).

Finally, since $\widetilde{X}$ is intended to model the pre-default prices of primary assets, we set $X_{t}=$ $\mathbb{1}_{\left\{t<\tau_{d}\right\}} \widetilde{X}_{t}$. Let us observe that $\widetilde{X}_{\cdot \wedge \tau_{d}}$ is a $\mathbb{G}$-semimartingale (since $\tau_{d}$ is an $\mathbb{F}$ - pseudo-stopping time, cf. Section 2.1p, and thus $X$ is an $\mathbb{R}^{d}$-valued, $\mathbb{G}$-semimartingale on $[0, T]$, which is null on $[0, T] \cap\left[\tau_{d}, \infty\right)$. The last feature reflects the fact that any value at $\tau_{d}$ is embedded in the recovery part of the dividend process $\mathcal{D}$ for $X$, given as

$$
\mathcal{D}_{t}=\int_{[0, t]}\left(1-H_{u}\right) d \mathcal{C}_{u}+\mathcal{R}_{u} d H_{u}
$$

We further define, for $t \in[0, T]$, the pre-default cumulative dividend process

$$
\overline{\mathcal{D}}_{t}=\int_{0}^{t} d \mathcal{C}_{u}+\mathcal{R}_{u} d \Gamma_{u}
$$

the credit-risk adjusted discount factor $\alpha_{t}=\exp \left(-\int_{0}^{t} \mu_{u} d u\right)$ with $\mu=r+\gamma$, and the pre-default cumulative price $\bar{X}$

$$
\bar{X}_{t}=\widetilde{X}_{t}+\alpha_{t}^{-1} \int_{0}^{t} \alpha_{u} d \overline{\mathcal{D}}_{u}
$$

Finally, we define the cumulative price $\widehat{X}$ by setting, for $t \in[0, T]$,

$$
\beta_{t} \widehat{X}_{t}=\mathbb{1}_{\left\{t<\tau_{d}\right\}} \beta_{t} \widetilde{X}_{t}+\int_{[0, t]} \beta_{u} d \mathcal{D}_{u}=\mathbb{1}_{\left\{t<\tau_{d}\right\}} \beta_{t}\left(\bar{X}_{t}-\alpha_{t}^{-1} \int_{0}^{t} \alpha_{u} d \overline{\mathcal{D}}_{u}\right)+\int_{[0, t]} \beta_{u} d \mathcal{D}_{u}
$$

The following result is the analog, relative to the primary market, of identity (15) for a game option. The proof is deferred to Appendix A.2.

Lemma 3.1 One has, for $t \in[0, T]$ :

$$
d\left(\beta_{t} \widehat{X}_{t}\right)=\mathbb{1}_{\left\{t \leq \tau_{d}\right\}} \beta_{t}\left(\alpha_{t}^{-1} d\left(\alpha_{t} \bar{X}_{t}\right)+\left(\mathcal{R}_{t}-\widetilde{X}_{t-}\right) d N_{t}^{d}\right) .
$$

In particular, if $\alpha \bar{X}$ is an $\mathbb{F}$ - local martingale, then $\beta \widehat{X}$ is a $\mathbb{G}$ - local martingale.

The situation considered in the following Corollary is common in applications (see, e.g., Proposition 4.2.

\footnotetext{
${ }^{2}$ As opposed to the case of a game option, we do not assume the variation of $\mathcal{C}$ to be bounded, in order to cover typical examples, see e.g. [5].
} 
Corollary 3.1 In the special case of a time-differentiable primary coupon process $\mathcal{C}=\int_{0}^{\cdot} c_{t} d t$ for an $\mathbb{F}$-adapted time-integrable coupon rate process $c$, and of a pre-default primary price process $\widetilde{X}$ with time-differentiable predictable finite variation component $\int_{0}^{*} x_{t} d t$ for some $\mathbb{F}$-adapted time-integrable process $x$, then $\beta \widehat{X}$ is a $\mathbb{G}$ - local martingale under the following arbitrage drift condition:

$$
x_{t}=\mu_{t} \widetilde{X}_{t}-c_{t}-\gamma_{t} \mathcal{R}_{t}, \quad t \in[0, T] .
$$

Proof. Given (23), we have

$$
d\left(\alpha_{t} \bar{X}_{t}\right)=d\left(\alpha_{t} \widetilde{X}_{t}\right)+\alpha_{t} d \overline{\mathcal{D}}_{t}=\alpha_{t}\left(d \widetilde{X}_{t}-\mu_{t} \widetilde{X}_{t} d t\right)+\alpha_{t} d \overline{\mathcal{D}}_{t} .
$$

In view of 22 and 26 , this implies that $\alpha \bar{X}$ is an $\mathbb{F}$ - local martingale, hence $\beta \hat{X}$ is a $\mathbb{G}$ - local martingale, by Lemma 3.1 .

The following proposition furnishes a generic constructive procedure for building arbitrage price processes for a primary market model.

Proposition 3.1 Let us be given a stochastic basis $(\Omega, \mathbb{F}, \mathbb{Q})$, an $\mathbb{F}$-adapted bounded from below and time-integrable process $r$, and an $\mathbb{F}$-adapted, non-negative and time-integrable process $\gamma$. Let a random time $\tau_{d}$ and filtrations $\mathbb{H}$ and $\mathbb{G}$ be defined by the canonical construction. In addition, let us be given an $\mathbb{R}^{d}$-valued, càdlàg $\mathbb{F}$ - special semimartingale $\widetilde{X}$ and an $\mathbb{R}^{d}$-valued, primary dividend process $\mathcal{D}$ as in (21), such that $\alpha \bar{X}$ is an $\mathbb{F}$ - local martingale. Then the discount factor $\beta_{t}=e^{-\int_{0}^{t} r_{u} d u}$ and the primary market risky price process $X_{t}=\mathbb{1}_{\left\{t<\tau_{d}\right\}} \tilde{X}_{t}$ define a primary market with arbitrage price process $X$, for any statistical probability $\mathbb{P} \sim \mathbb{Q}$.

Proof. Most of the proposition follows by construction of the model. The only point that requires a justification is that $X$ is an arbitrage price process. But this results from Lemma 3.1 which tells us that $\beta \widehat{X}$ is a $(\mathbb{G}, \mathbb{Q})$ - local martingale.

Henceforth a primary market arbitrage price process $X$ constructed in this way will be called a $(\Omega, \mathbb{F}, \mathbb{Q})$ - canonical intensity market model.

\subsection{Markovian BSDE}

The market model constructed above is too general to be suitable for practical purposes. In particular, for computational purposes, it is necessary to impose some Markovian structure on a market model. For a given valuation problem at hand, this will be achieved, by producing a pre-default factor model $\mathcal{Z}$ in which the related $\operatorname{BSDE}(\mathcal{E})$ is in fact a Markovian BSDE (see [8, 14]). If the primary market is also Markovian in some sense with respect to $\mathcal{Z}$, it will further be possible to provide and analyze explicit and computable hedging strategies.

Let us thus be given a (game) option with data $C, R, L, U, \xi, \bar{\tau}$, in a $(\Omega, \mathbb{F}, \mathbb{Q})$ - canonical intensity market model, and for a driver $F$ of the form $(10)$, for some $\mathbb{F}$-adapted, time-integrable process $f$ over $[0, T]$ (which will sometimes also be called the driver in the sequel), such that the process $\bar{F}-F$ is bounded from below. We are thus in the special case where the first line of $(\mathcal{E})$ can equivalently be rewritten as (11).

Definition 3.1 We say that the $\operatorname{BSDE}(\mathcal{E})$ is a Markovian BSDE, if:

- the input data $\mu=r+\gamma, f, \chi, \mathcal{L}$ and $\mathcal{U}$ of $(\mathcal{E})$ (with the first line of $(\mathcal{E})$ represented by (11)) are given by Borel-measurable functions of an $(\Omega, \mathbb{F}, \mathbb{Q}$ )-Markov process $\mathcal{Z}$ taking values in a finitedimensional Borel state space $E$ (with first component given by time $t$ ), $\mathrm{sc}^{3}$

$$
\begin{gathered}
r_{t}=r\left(\mathcal{Z}_{t}\right), \gamma_{t}=\gamma\left(\mathcal{Z}_{t}\right) \\
f_{t}=f\left(\mathcal{Z}_{t}\right), \chi=\chi\left(\mathcal{Z}_{T}\right), \mathcal{L}_{t}=\mathcal{L}\left(\mathcal{Z}_{t}\right), \mathcal{U}_{t}=\mathcal{U}\left(\mathcal{Z}_{t}\right) ;
\end{gathered}
$$

\footnotetext{
${ }^{3}$ The related functions are denoted by the same symbols as the corresponding processes.
} 
- $\bar{\tau}$ is the first time of entry (capped at $T$ ) by the process $\mathcal{Z}$ into a given closed subset of $E$.

In particular, the system made of the specification of forward dynamics for $\mathcal{Z}$ and of the BSDE $(\mathcal{E})$ constitutes a decoupled Markovian FBSDE in $(\mathcal{Z}, \Theta, M, K)$. This equation is decoupled, in the sense that the forward component of the system serves as an input for the backward component (i.e., $\mathcal{Z}$ is an input to $(\mathcal{E})$, cf. (27)), but not the other way round.

Of course, the possibility of finding such a process $\mathcal{Z}$ and the nature of $\mathcal{Z}$ obviously depend on the driver $f$ in $(\mathcal{E})$, so the following developments are, once again, parameterized by the choice of the process $f$ in 10 (see Section 4 for a concrete example, Subsection 4.4 in particular).

From the point of view of the financial interpretation, the components of $\mathcal{Z}$ are observable factors. The first component of $\mathcal{Z}$ (indexed by 0 ) is simply time $\mathcal{Z}_{t}^{0}=t$. The remaining components of $\mathcal{Z}$ are intimately, though non-trivially, connected to the pre-default price process $\widetilde{X}$ as follows:

- Most components of $\widetilde{X}$ will usually be given by some components of $\mathcal{Z}$. Note, however, that typically there will be some primary risky assets in $X$, introduced for hedging purposes, which are not represented in $\mathcal{Z}$. In particular one 'extra asset' is typically required for hedging default risk (if wished), which is not (explicitly, at least) present in the filtration $\mathbb{F}$ of $\mathcal{Z}$;

- The components of $\mathcal{Z}$ that are not included in $\widetilde{X}$ (if any) are to be understood as simple factors that may be required to 'Markovianize' the payoffs of a game option (e.g., factors accounting for path dependence in the option's payoff and/or non-traded factors such as stochastic volatility in the dynamics of the assets underlying the option);

- There exists a well-defined and constructive mapping from a collection of meaningful and 'directly observable' economic variables to $\mathcal{Z}$. Note that, due to the nature of the model, the observability of the factor process $\mathcal{Z}$ in the mathematical sense of $\mathbb{F}$-adaptedness is not sufficient in practice. For a model to be implementable, a constructive mapping from a collection of meaningful and directly observable economic variables to $\mathcal{Z}$ is really needed. Otherwise, the model would be in fact useless.

\subsection{Jump-Diffusion Setting with Regimes}

Under a rather generic specification for the Markov factor process $\mathcal{Z}$, we shall now derive the associated Markovian BSDE, as well as the related obstacles problem, that is, a coupled system of partial integro-differential variational inequalities.

To this end, given an integer $p$ and a finite set $I$ with $l$ elements, we define the following linear operator $\mathcal{A}$ acting on regular function $\bigcup^{4} \Theta=\Theta(t, x, y)$, for $(t, x, y) \in E=[0, T] \times \mathbb{R}^{p} \times I$ :

$$
\begin{aligned}
& \mathcal{A} \Theta(t, x, y)=\frac{1}{2} \sum_{i, j=1}^{p} a_{i j}(t, x, y) \partial_{x_{i} x_{j}}^{2} \Theta(t, x, y) \\
& \quad+\sum_{i=1}^{p}\left(b_{i}(t, x, y)-g(t, x, y) \int_{\mathbb{R}^{p}} u_{i}\left(t, x, y, x^{\prime}\right) h\left(t, x, y, d x^{\prime}\right)\right) \partial_{x_{i}} \Theta(t, x, y) \\
& \quad+g(t, x, y) \int_{\mathbb{R}^{p}}\left(\Theta\left(t, x+u\left(t, x, y, x^{\prime}\right), y\right)-\Theta(t, x, y)\right) h\left(t, x, y, d x^{\prime}\right) \\
& \quad+\sum_{y^{\prime} \in I} \lambda\left(t, x, y, y^{\prime}\right)\left(\Theta\left(t, x, y^{\prime}\right)-\Theta(t, x, y)\right),
\end{aligned}
$$

where:

- the $a(t, x, y)$ are $p$-dimensional covariance matrices, with $a(t, x, y)=\sigma(t, x, y) \sigma(t, x, y)^{\mathrm{\top}}$, for some $p$-dimensional dispersion matrices $\sigma(t, x, y)$;

- the $b(t, x, y)$ are $p$-dimensional drift vector coefficients;

\footnotetext{
${ }^{4}$ We use the same notation $\Theta$ for the state-process of a solution to a BSDE and for a generic function devoted to represent the solution of a related PIDE, cf. 34.
} 
- the jump intensity function $g(t, x, y)$ is non-negative, the $h(t, x, y, \cdot)$ are probability measures on $\mathbb{R}^{p}$, and $u\left(t, x, y, x^{\prime}\right)$ is the jump size function,;

- the intensity matrix function $\left[\lambda\left(t, x, y, y^{\prime}\right)\right]_{y, y^{\prime} \in I}$ is such that $\lambda\left(t, x, y, y^{\prime}\right) \geq 0$ whenever $y \neq y^{\prime}$, and $\lambda(t, x, y, y)=-\sum_{y^{\prime} \in I \backslash\{y\}} \lambda\left(t, x, y, y^{\prime}\right)$.

Under appropriate technical conditions (see 8] for a detailed set of explicit conditions, or see Theorems 4.1 and 5.4 in Chapter 4 of Ethier and Kurtz [15] for abstract conditions regarding the existence and uniqueness of a solution to the related martingale problem with generator $\mathcal{A}$ ), there exists a stochastic basis $(\Omega, \mathbb{F}, \mathbb{Q})$ on $[0, T]$, endowed with a $p$-dimensional Brownian motion $W$, an integer-valued random measure $J$ (see Jacod and Shiryaev [18, Definition II.1.13 p.68]), and an $(\Omega, \mathbb{F}, \mathbb{Q})$-Markov càdlàg process $\mathcal{Z}=(t, \mathcal{X}, \mathcal{Y})$, such that:

- The $\mathbb{Q}$-compensated martingale measure $\widetilde{\nu}$ of the integer-valued random measure $\nu$ on $I$ which counts the transitions $\nu_{t}(y)$ of $\mathcal{Y}$ to state $y$ between time 0 and time $t$, is given by

$$
d \widetilde{\nu}_{t}(y)=d \nu_{t}(y)-\mathbb{1}_{\left\{\mathcal{Y}_{t} \neq y\right\}} \lambda\left(t, \mathcal{X}_{t}, \mathcal{Y}_{t}, y\right) d t
$$

Hence $\mathcal{Y}$ admits the following special semimartingale canonical representation:

$$
d \mathcal{Y}_{t}=\sum_{y \in I} \lambda\left(t, \mathcal{X}_{t}, \mathcal{Y}_{t}, y\right)\left(y-\mathcal{Y}_{t}\right) d t+\sum_{y \in I}\left(y-\mathcal{Y}_{t-}\right) d \widetilde{\nu}_{t}(y), t \in[0, T]
$$

- The $\mathbb{Q}$-compensated martingale (random) measure $\widetilde{J}$ of $J$ is given by

$$
\widetilde{J}(d t, d x)=J(d t, d x)-g\left(t, \mathcal{X}_{t}, \mathcal{Y}_{t}\right) h\left(t, \mathcal{X}_{t}, \mathcal{Y}_{t}, d x\right) d t
$$

and the $\mathbb{R}^{p}$-valued process $\mathcal{X}$ satisfies, for $t \in[0, T]$,

$$
d \mathcal{X}_{t}=b\left(t, \mathcal{X}_{t}, \mathcal{Y}_{t}\right) d t+\sigma\left(t, \mathcal{X}_{t}, \mathcal{Y}_{t}\right) d W_{t}+\int_{\mathbb{R}^{p}} u\left(t, \mathcal{X}_{t-}, \mathcal{Y}_{t-}, x\right) \widetilde{J}(d x, d t)
$$

In particular, we then have the following variant of the Itô formula (see, e.g., Jacod [17, Theorem 3.89 p.109]), in which $\partial$ denotes the row-gradient of $\Theta(t, x, y)$ with respect to $x$ :

$$
\begin{aligned}
& d \Theta\left(\mathcal{Z}_{t}\right)=\left(\partial_{t}+\mathcal{A}\right) \Theta\left(\mathcal{Z}_{t}\right) d t+\partial \Theta\left(\mathcal{Z}_{t}\right) \sigma\left(\mathcal{Z}_{t}\right) d W_{t} \\
& \quad+\int_{\mathbb{R}^{p}}\left(\Theta\left(t, \mathcal{X}_{t-}+u\left(\mathcal{Z}_{t-}, x\right), \mathcal{Y}_{t-}\right)-\Theta\left(\mathcal{Z}_{t-}\right)\right) \widetilde{J}(d x, d t) \\
& \quad+\sum_{y \in I}\left(\Theta\left(t, \mathcal{X}_{t-}, y\right)-\Theta\left(\mathcal{Z}_{t-}\right)\right) d \widetilde{\nu}_{t}(y)
\end{aligned}
$$

which holds for a sufficiently regular function $\Theta$.

Remarks 3.1 (i) If we suppose that the intensity matrix of $\mathcal{Y}$ does not depend on $t, x$, then $\mathcal{Y}$ is a continuous time Markov chain with finite state space $I$. Alternatively, if we take $g\left(t, x, y, x^{\prime}\right)=x^{\prime}$, and we suppose that the coefficients $\sigma, b, u, g$ and $h$ do not depend on $t, x, y$, then $\mathcal{X}$ is a Lévy-Poisson process. In general, we deal with a $\mathcal{Y}$-modulated Lévy-like component $\mathcal{X}$ and an $\mathcal{X}$-modulated Markov chain-like component $\mathcal{Y}$. For simplicity we do not consider the 'infinite activity' case, that is, the case when the jump measure of $\mathcal{X}$ is unbounded. Note however that our approach could be extended to the infinite activity without major changes if wished. $\mathcal{Z}$ thus defines a rather general class of Markov processes to be used as factor processes in financial modeling.

(ii) From the point of view of interpretation, process $\mathcal{Y}$ represents regimes that modulate the dynamics of the risk-neutral pricing process. In order to make the calibration of such a risk-neutral pre-default model possible, various regimes $y \in I$ should correspond to non-overlapping (vectorvalued) sets of model parameters.

Remarks 3.2 For $l=1$, that is, in the case when the regime indicator process is constant, the one-dimensional process $\widetilde{\nu}$ in $\sqrt{29})$ is trivially null and plays no role whatsoever, so that we may and do redefine $l$ as 0 (see Section 4.3 for a concrete example). 


\subsection{Variational Inequality Approach}

Given such a factor process $\mathcal{Z}$ and suitable Borel functions $r$ and $\gamma$, the related stochastic basis $(\Omega, \mathbb{F}, \mathbb{Q})$ and processes $r_{t}=r\left(\mathcal{Z}_{t}\right), \gamma_{t}=\gamma\left(\mathcal{Z}_{t}\right)$ can be used as starting points in the construction of a canonical intensity model with respect to $(\Omega, \mathbb{F}, \mathbb{Q})$ (cf. Proposition 3.1). Denote by $\mathcal{P}$ the $\mathbb{F}$-predictable $\sigma$-algebra on $\Omega \times[0, T]$ and by $\mathcal{B}\left(\mathbb{R}^{p}\right)$ the Borel $\sigma$-algebra on $\mathbb{R}^{p}$. Given a further primary dividend process $\mathcal{D}$, process $\widetilde{X}$ may thus be defined as $\widetilde{X}=\bar{X}-\alpha_{t}^{-1} \int_{0}^{t} \alpha_{u} d \overline{\mathcal{D}}_{u}$ (cf. 23 ), where, consistently with arbitrage requirements (cf. Lemma 3.1), the pre-default cumulative price $\bar{X}$ is defined over $[0, T]$ by $\bar{X}_{0}=0$ and

$$
\alpha_{t}^{-1} d\left(\alpha_{t} \bar{X}_{t}\right)=\widetilde{Z}_{t} d W_{t}+\widetilde{Y}_{t} d \widetilde{\nu}_{t}+\int_{\mathbb{R}^{p}} \widetilde{V}_{t}(x) \widetilde{J}(d x, d t)
$$

for $\mathbb{R}^{d \otimes p}$-valued and $\mathbb{R}^{d \otimes l} \mathbb{F}$-predictable processes $\widetilde{Z}$ and $\widetilde{Y}$, and for a $d$-valued vector $\widetilde{V}$ of $\mathcal{P} \otimes \mathcal{B}\left(\mathbb{R}^{p}\right)$ measurable random functions. In order for the (fully decoupled and explicit) Markovian SDE (32) to define a well-posed problem, we impose the following integrability conditions, denoting $I=$ $\left\{y^{1}, \ldots, y^{l}\right\}$ (with the convention that $l=0$ when there are no regimes, or, equivalently, only one regime, in the model, cf. Remark 3.2):

$$
\begin{gathered}
\sum_{i=1}^{d} \sum_{j=1}^{p} \mathbb{E}_{\mathbb{Q}}\left(\int_{0}^{T}\left(\widetilde{Z}_{t}^{i, j}\right)^{2} d t \mid \mathcal{F}_{0}\right)+\sum_{i=1}^{d} \sum_{j=1}^{l} \mathbb{E}_{\mathbb{Q}}\left(\int_{0}^{T}\left(\widetilde{Y}_{t}^{i, j}\right)^{2} \lambda\left(\mathcal{Z}_{t}, y^{j}\right) d t \mid \mathcal{F}_{0}\right)<\infty, \quad \text { a.s. } \\
\sum_{i=1}^{d} \mathbb{E}_{\mathbb{Q}}\left(\int_{0}^{T} \int_{\mathbb{R}^{p}}\left(\widetilde{V}_{t}^{i}\right)^{2}(x) g\left(\mathcal{Z}_{t}\right) h\left(\mathcal{Z}_{t}, d x\right) d t \mid \mathcal{F}_{0}\right)<\infty, \quad \text { a.s. }
\end{gathered}
$$

Hence well-posedness of the (fully decoupled and explicit) Markovian SDE (32) follows.

Remarks 3.3 The standing example in which 32 holds along with all the required conditions is given by the typical situation where $\widetilde{X}_{t}=\tilde{X}\left(\mathcal{Z}_{t}\right)$ for a Borel-measurable function $\tilde{X}$ with (at least) the same regularity as the function $\Theta$ to be introduced in Theorem 3.1, see further developments following Theorem 3.1 .

Having introduced a further Markovian $\operatorname{BSDE}(\mathcal{E})$, a solution $(\Theta, M, K)$ to $(\mathcal{E})$ is then typically sought for with $M$ in the form

$$
M_{t}=\int_{0}^{t} Z_{u} d W_{u}+\int_{0}^{t} Y_{u} d \widetilde{\nu}_{u}+\int_{0}^{t} \int_{\mathbb{R}^{p}} V_{u}(x) \widetilde{J}(d x, d u)
$$

for $\mathbb{F}$-predictable processes $Z, Y$ and a $\mathcal{P} \otimes \mathcal{B}\left(\mathbb{R}^{p}\right)$-measurable random function $V: \Omega \times[0, T] \times \mathbb{R}^{p} \rightarrow \mathbb{R}$ such that:

$$
\begin{gathered}
\sum_{j=1}^{p} \mathbb{E}_{\mathbb{Q}}\left(\int_{0}^{T}\left(Z_{t}^{j}\right)^{2} d t \mid \mathcal{F}_{0}\right)+\sum_{j=1}^{l} \mathbb{E}_{\mathbb{Q}}\left(\int_{0}^{T}\left(Y_{t}^{j}\right)^{2} \lambda\left(t, \mathcal{X}_{t}, \mathcal{Y}_{t}, y^{j}\right) d t \mid \mathcal{F}_{0}\right)<\infty, \quad \text { a.s. } \\
\mathbb{E}_{\mathbb{Q}}\left(\int_{0}^{T} \int_{\mathbb{R}^{p}} V_{t}^{2}(x) g\left(t, \mathcal{X}_{t}, \mathcal{Y}_{t}\right) h\left(t, \mathcal{X}_{t}, \mathcal{Y}_{t}, d x\right) d t \mid \mathcal{F}_{0}\right)<\infty, \quad \text { a.s. }
\end{gathered}
$$

We are thus led to look for solutions $(\Theta, M, K)$ to $(\mathcal{E})$ with $M$ in the form $(33)$, where $Z, Y$ and $V$ are parts of a solution. We thus get a Markovian BSDE in the unknowns $(\Theta, Z, Y, V, K)$.

In particular, it is shown in [10] that, under mild regularity conditions, $(\mathcal{E})$ has a unique solution $(\Theta, M, K)$ with $M$ of the form [33), in suitable Hilbert spaces. In the Markovian case, [8] establishes the relation between this solution and the unique solution in some sense (viscosity solution with polynomial growth in the $x$ variable), $\Theta(t, x, y)$, to an associated PIDE obstacle problem. In the simplest case where $\bar{\tau}=0$ (that is, no call protection), the associated obstacles problem is given by the following system of $l$ coupled partial integro-differential variational inequalities in spacedimension $p$ :

$$
\begin{aligned}
& \max \left(\operatorname { m i n } \left(-\partial_{t} \Theta(t, x, y)-\mathcal{A} \Theta(t, x, y)-f(t, x, y)+\mu(t, x, y) \Theta(t, x, y),\right.\right. \\
&\Theta(t, x, y)-\mathcal{L}(t, x, y)), \Theta(t, x, y)-\mathcal{U}(t, x, y))=0, \quad t<T,(x, y) \in \mathbb{R}^{p} \times I,
\end{aligned}
$$

with terminal condition $\Theta(T, x, y)=\chi(x, y)$. So, 
Theorem 3.1 Under mild conditions, one has, for $t \in[0, T]$ :

$$
\Theta_{t}=\Theta\left(\mathcal{Z}_{t}\right)
$$

Moreover, in regular cases, we also have in some sense (see [9]), for $t \in[0, T]$ :

$$
\begin{gathered}
Z_{t}=\partial \Theta \sigma\left(\mathcal{Z}_{t}\right) \\
Y_{t}=\Theta\left(t, \mathcal{X}_{t-}\right)-\Theta\left(\mathcal{Z}_{t-}\right) \\
V_{t}(\cdot)=\Theta\left(t, \mathcal{X}_{t-}+u\left(\mathcal{Z}_{t-}, \cdot\right), \mathcal{Y}_{t-}\right)-\Theta\left(\mathcal{Z}_{t-}\right)
\end{gathered}
$$

where $\Theta(t, x)$ stands for $\left(\Theta\left(t, x, y^{1}\right), \cdots, \Theta\left(t, x, y^{l}\right)\right)$ in the second line.

Let us further assume that the primary pre-default price $\widetilde{X}$ satisfies likewise $\widetilde{X}_{t}=\widetilde{X}\left(\mathcal{Z}_{t}\right)$ for a function $\widetilde{X}$ with the same regularity as $\Theta$, and that, consistently with 32 :

$$
\begin{aligned}
& \alpha_{t}^{-1} d\left(\alpha_{t} \bar{X}_{t}\right)=\partial \widetilde{X} \sigma\left(\mathcal{Z}_{t}\right) d W_{t}+ \\
& \quad\left[\widetilde{X}\left(t, \mathcal{X}_{t-}\right)-\widetilde{X}\left(\mathcal{Z}_{t-}\right)\right] d \widetilde{\nu}_{t}+\int_{\mathbb{R}^{p}}\left[\widetilde{X}\left(t, \mathcal{X}_{t-}+u\left(\mathcal{Z}_{t-}, x\right), \mathcal{Y}_{t-}\right)-\widetilde{X}\left(\mathcal{Z}_{t-}\right)\right] \widetilde{J}(d x, d t)
\end{aligned}
$$

We thus get a pre-default cumulative primary market $\mathbb{Q}$-value dynamics for $\bar{X}$ of the form $(32)$, with processes $\widetilde{Z}, \widetilde{Y}$ and random measure $\widetilde{V}$ therein given by, for $t \in[0, T]$ :

$$
\begin{gathered}
\widetilde{Z}_{t}=\partial \widetilde{X} \sigma\left(\mathcal{Z}_{t}\right) \\
\widetilde{Y}_{t}=\widetilde{X}\left(t, \mathcal{X}_{t-}\right)-\widetilde{X}\left(\mathcal{Z}_{t}\right) \\
\widetilde{V}_{t}(\cdot)=\widetilde{X}\left(t, \mathcal{X}_{t-}+u\left(\mathcal{Z}_{t-}, \cdot\right), \mathcal{Y}_{t-}\right)-\widetilde{X}\left(\mathcal{Z}_{t-}\right)
\end{gathered}
$$

where $\widetilde{X}(t, x)$ stands for $\left(\widetilde{X}\left(t, x, y^{1}\right), \cdots, \widetilde{X}\left(t, x, y^{l}\right)\right)$ in the second line. Given 15,25 and 32 , the cost $\rho=\rho(\zeta)$ relative to the strategy $\zeta$ (cf. (16)) can in turn be expressed in terms of the pricing functions $\Theta$ and $\widetilde{X}$ and the related delta functions.

Theorem 3.2 In the Markovian set-up, the dynamics (16) for the cost process $\rho$ relative to the strategy $\zeta$ (and thus the related profit and loss process e, cf. [18)) may be rewritten as

$$
\begin{aligned}
d \rho_{t}=\mathbb{1}_{\left\{t \leq \tau_{d}\right\}} \beta_{t} & \left(\left(Z_{t}-\zeta_{t} \widetilde{Z}_{t}\right) d W_{t}+\left(Y_{t}-\zeta_{t} \widetilde{Y}_{t}\right) d \widetilde{\nu}_{t}\right. \\
& \left.+\int_{\mathbb{R}^{p}}\left(V_{t}(x)-\zeta_{t} \widetilde{V}_{t}(x)\right) \widetilde{J}(d x, d t)+\left(\left(R_{t}-\widetilde{\Pi}_{t-}\right)-\zeta_{t}\left(\mathcal{R}_{t}-\widetilde{X}_{t-}\right) d N_{t}^{d}\right)\right)
\end{aligned}
$$

or more specifically, in regular cases where identities (35) and (37) apply:

$$
\begin{aligned}
d \rho_{t}=\mathbb{1}_{\left\{t \leq \tau_{d}\right\}} \beta_{t} & \left(\left(\partial \Theta \sigma\left(\mathcal{Z}_{t}\right)-\zeta_{t} \partial \widetilde{X} \sigma\left(\mathcal{Z}_{t}\right)\right) d W_{t}\right. \\
& +\left(\left[\Theta\left(t, \mathcal{X}_{t-}\right)-\Theta\left(\mathcal{Z}_{t-}\right)\right]-\zeta_{t}\left[\widetilde{X}\left(t, \mathcal{X}_{t-}\right)-\widetilde{X}\left(\mathcal{Z}_{t-}\right)\right]\right) d \widetilde{\nu}_{t} \\
& +\int_{\mathbb{R}^{p}}\left(\left[\Theta\left(t, \mathcal{X}_{t-}+u\left(\mathcal{Z}_{t-}, x\right), \mathcal{Y}_{t-}\right)-\Theta\left(\mathcal{Z}_{t-}\right)\right]\right. \\
& \left.\quad-\zeta_{t}\left[\widetilde{X}\left(t, \mathcal{X}_{t-}+u\left(\mathcal{Z}_{t-}, x\right), \mathcal{Y}_{t-}\right)-\widetilde{X}\left(\mathcal{Z}_{t-}\right)\right]\right) \widetilde{J}(d x, d t) \\
& \left.+\left(\left(R_{t}-\widetilde{\Pi}_{t-}\right)-\zeta_{t}\left(\mathcal{R}_{t}-\widetilde{X}_{t-}\right) d N_{t}^{d}\right)\right)
\end{aligned}
$$

Remarks 3.4 (i) Provided related matrices are left-invertible over $\left[0, T \wedge \tau_{d}\right]$ (which means that the primary market is sufficiently rich), it is thus possible to hedge completely the source risks $W$, $\nu$ and $H$, or any finite subset of their components. Note that the strategy consisting (under the related left-invertibility assumption) to hedge a given set of components of $W, \nu$ and/or $H$ on one hand, on the other hand creates some risk via the dependence wrt $\zeta$ of the remaining terms in (38). 
(ii) Of course a perfect hedge $(\rho=0)$ is hopelesss unless there are no jumps (or only a finite number of jump sizes) in $\mathcal{X}$. In the context of incomplete markets the choice of a hedging strategy is up to one's optimality criterion relative to the hedging cost $(16)-(38)$. For instance, a trader may wish to minimize the (objective, $\mathbb{P}-$ ) variance of $\int_{0}^{T} \beta_{t} d \rho_{t}$. Yet the related strategy $\widehat{\zeta}^{v a}$ is hardly accessible in practice (in particular it typically depends on the objective model drift, a quantity notoriously difficult to estimate on financial data). As a proxy to this strategy, traders commonly use the strategy $\zeta^{v a}$ which locally minimizes the risk-neutral variance of the error. In view of $(16)$, the related strategy is given as the solution of the linear regression problem of $\beta d M_{t}$ against $d\left(\beta_{t} \widehat{X}_{t}\right)$, so, formally:

$$
\zeta_{t}^{v a}=\operatorname{Cov}_{t}\left(d\left(\beta_{t} \widehat{X}_{t}\right)\right)^{-1} \operatorname{Cov}_{t}\left(d\left(\beta_{t} \widehat{X}_{t}\right), \beta d M_{t}\right)
$$

with $\operatorname{Cov}_{t}\left(d A_{t}, d B_{t}\right):=\frac{1}{h} \lim _{h \rightarrow 0} \operatorname{Cov}_{t}\left(A_{t+h}-A_{t}, B_{t+h}-B_{t}\right)$. In the context of a specific Markovian model it is then often easy to derive an explicit formula for 40).

\section{Study of a Defaultable Convertible Bonds}

In the concluding part of this paper, we shall apply the results of the previous sections to the case of a defaultable convertible bond with the underlying $S$, one of the primary risky assets.

\subsection{Specification of the Payoffs}

To describe the covenants of a typical convertible bond (CB), we need to introduce some additional notation:

$\bar{N}$ : the par (nominal) value,

$S$ : the price process of the asset underlying the $\mathrm{CB}$,

$\widetilde{S}$ : the pre-default value process of $S$,

$c^{c b}$ : the continuous coupon rate process; a bounded, $\mathbb{F}$ - progressively measurable process,

$T_{i}, c^{i}, i=0,1, \ldots, K\left(T_{0}=c^{0}=0\right)$ : discrete coupon dates and amounts; the discrete coupon dates $T_{0}, \ldots, T_{K}$ are deterministic fixed times with $T_{K-1}<T \leq T_{K}$; the discrete coupon amounts $c^{i}$ are bounded, $\mathcal{F}_{T_{i-1}}$-measurable random variables, for $i=1,2, \ldots, K$,

$A_{t}$ : the accrued interest at time $t$, specifically,

$$
A_{t}=\frac{t-T_{i_{t}-1}}{T_{i_{t}}-T_{i_{t}-1}} c^{i_{t}}
$$

where $i_{t}$ is the integer satisfying $T_{i_{t}-1} \leq t<T_{i_{t}}$; in view of our assumptions, the process $A$ is an $\mathbb{F}$-adapted, càdlàg process with finite variation.

$\bar{R}$ : the recovery process on the $\mathrm{CB}$ upon default of the issuer; an $\mathbb{F}$-predictable, bounded process,

$\kappa:$ the bond's conversion factor,

$\bar{P} \leq \bar{C}$ : the put and call nominal payments, respectively; by assumption $\bar{P} \leq \bar{N} \leq \bar{C}$.

For a more detailed description of covenants of convertible bonds, the interested reader is referred to [3]. Let us only mention that a real-life convertible bonds typically includes a positive call notice period so that they may continue to live some time beyond the call time $\tau_{c}$. A convertible bond with positive call notice period is thus a contract that becomes an American option upon call. In particular, a convertible bond with positive call notice period does not strictly fit the definition 2.1 of a game option (precise modeling involves three stopping times, see [3]). 
To circumvent this difficulty, we developed in $[3]$ an approach consisting in treating such a product as a contract that pays upon call the value of an American option. At least in complete markets or, more generally, whenever arbitrage prices of defaultable American options are unambiguously defined, this interpretation seems to be reasonable. Note that for practical purposes one can often 'complete the market', in the sense that arbitrage prices of defaultable game options become uniquely defined in the completed market (see, for instance, [5]).

To sum up, we propose in [3] a recursive approach to the valuation of a $\mathrm{CB}$ with positive call notice period. In the first step, a CB is valued upon call. Subsequently, we use the resulting price upon call as the payoff at call of a CB without call notice period. In this way, a CB with positive call notice period can be priced as a reduced convertible bond, which is formally defined as follows.

Definition 4.1 A reduced convertible bond is a game option with coupon process $C$, recovery process $R^{c b}$ and payoffs $L^{c b}, U^{c b}, \xi^{c b}$ such that

$$
\begin{gathered}
C_{t}=\int_{0}^{t} c_{u}^{c b} d u+\sum_{0 \leq T_{i} \leq t} c^{i}, \quad R_{t}^{c b}=(1-\eta) \kappa \widetilde{S}_{t-} \vee \bar{R}_{t}, \\
L_{t}^{c b}=\bar{P} \vee \kappa \widetilde{S}_{t}+A_{t}, \quad \xi^{c b}=\bar{N} \vee \kappa \widetilde{S}_{T}+A_{T},
\end{gathered}
$$

and where $\left(U_{t}^{c b}\right)_{t \in[0, T]}$ is some $\mathbb{F}$-adapted càdlàg process satisfying the following inequality:

$$
\bar{C} \vee \kappa \widetilde{S}_{t}+A_{t} \leq U_{t}^{c b}, \quad t \in[0, T]
$$

In the financial interpretation, $U_{t}^{c b}$ represents the pre-default value of the reduced convertible bond upon a call at time $t$. In particular, a convertible bond without call notice period is a reduced convertible bond with $U_{t}^{c b}=\bar{C} \vee \kappa \widetilde{S}_{t}+A_{t}$ for $t \in[0, T]$. Note that under our assumption that $\bar{P} \leq \bar{N} \leq \bar{C}$, we obtain by 41 :

$$
L_{T}^{c b}=\bar{P} \vee \kappa \widetilde{S}_{T}+A_{T} \leq \bar{N} \vee \kappa \widetilde{S}_{T}+A_{T}=\xi^{c b} \leq \bar{C} \vee \kappa \widetilde{S}_{T}+A_{T} \leq U_{T}^{c b}
$$

\subsection{Clean Price}

In the sequel, we shall focus on reduced convertible bonds.

Definition 4.2 For a pre-default $\mathbb{Q}$-price $\widetilde{\Pi}$ of a (reduced) convertible bond, by the clean price of this bond we mean the difference $\widetilde{\Pi}-A$.

The notion of the clean price is consistent with the market convention for bonds, which relies on subtracting the accrued interest from the trading (dirty) price. Market quotations for bonds are usually given in terms of clean prices (or bond yields), in order to avoid coupon-related discontinuities in quotations.

Let us set $a_{t}=\frac{c^{i_{t}}}{T_{i_{t}}-T_{i_{t}}-1}$ for $t \in[0, T]$. Then

$$
A_{t}=\int_{0}^{t} a_{u} d u-\sum_{0 \leq T_{i} \leq t} c^{i}
$$

and the integration by part formula yields (recall that $B=-\ln \alpha$ ):

$$
\alpha_{t} A_{t}=\int_{0}^{t} \alpha_{u}\left(a_{u} d u-A_{u} d B_{u}\right)-\sum_{0 \leq T_{i} \leq t} \alpha_{T_{i}} c^{i} .
$$

Let us fix some risk-neutral measure $\mathbb{Q}$. The motivation for the choice of the driver $F$ defined by 43 in Propositions 4.14 .2 will be discussed in Subsection 4.4 
Proposition 4.1 (i) Considering a reduced convertible bond, let us choose the driver

$$
F^{c b}:=\bar{F}+A,
$$

where $\bar{F}$ was defined in (8). Then the data of the doubly reflected BSDE (E) take the following form:

$$
\begin{gathered}
F^{c b}, \chi=\xi^{c b}-A_{T}=\bar{N} \vee \kappa \widetilde{S}_{T}, \\
\mathcal{L}=L^{c b}-A=\bar{P} \vee \kappa \widetilde{S}, \mathcal{U}=U^{c b}-A, \bar{\tau} .
\end{gathered}
$$

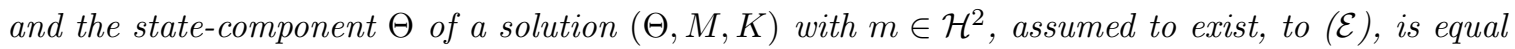
to the clean price of the bond.

(ii) In the default intensity set-up, $F^{c b}$ is of the form (10) with

$$
f=\gamma R^{c b}+c^{c b}+a-\mu A=: f^{c b} .
$$

(iii) Assume that the pre-default value process $\widetilde{S}$ is continuous. Then the lower barrier process $\mathcal{L}$ is continuous on $[0, T]$. Moreover, in the case of a convertible bond with no call notice period, $\mathcal{U}$ is continuous on $[0, T]$, and the upper barrier process $\overline{\mathcal{U}}$ is continuous on $[\bar{\tau}, T]$.

Proof. (i) We have, by 12,

$$
\Theta=\widetilde{\Pi}+\bar{F}-F^{c b}=\widetilde{\Pi}-A,
$$

in view of the definition of $F^{c b}$. Also,

$$
\mathcal{L}=L^{c b}+\bar{F}-(\bar{F}+A)=L^{c b}-A .
$$

The other identities can be shown similarly.

(ii) Using the definition of $f^{c b}$ and $\sqrt{42}$ with $d B_{u}=\mu_{u} d u$ in an intensity default model, we get:

$$
\begin{gathered}
\int_{0}^{t} \alpha_{u} f_{u}^{c b} d u=\int_{0}^{t} \alpha_{u} \gamma_{u} R_{u}^{c b} d u+\int_{0}^{t} \alpha_{u} c_{u}^{c b} d u+\int_{0}^{t} \alpha_{u}\left(a_{u}-\mu_{u} A_{u}\right) d u \\
=\alpha_{t} A_{t}+\int_{0}^{t} \alpha_{u} d C_{u}+\int_{0}^{t} \alpha_{u} \gamma_{u} R_{u}^{c b} d u=\alpha_{t}\left(A_{t}+\bar{F}_{t}\right)=\alpha_{t} F_{t}^{c b} .
\end{gathered}
$$

(iii) It suffices to note that for a convertible bond with no call notice period we have $\mathcal{U}_{t}=U_{t}^{c b}-A_{t}=$ $\bar{C} \vee \kappa \widetilde{S}_{t}$, and, for $t \geq \bar{\tau}, \overline{\mathcal{U}}_{t}=\mathcal{U}_{t}$.

Let us summarize our findings at this point of this section. First, we have shown that by solving the doubly reflected BSDE $(\mathcal{E})$ with the driver $F=F^{c b}$ given by 44 , we obtain the clean price of a reduced convertible bond as the state process $\Theta$ of a solution to $(\mathcal{E})$.

Second, the related driver terms in $(\mathcal{E})$ are then given as integrals with respect to the Lebesgue measure, which is the standard form in the BSDE literature.

Third, under mild assumptions, the lower and upper barriers for this choice of the driver $F$ are given as continuous processes (at least, in case with no notice period; see Subsection 4.4 for more about this), and thus the state process process $\Theta$ of a solution to the doubly reflected $\operatorname{BSDE}(\mathcal{E})$ is continuous, provided the $\mathbb{F}$-martingale $M$ is continuous.

\subsection{A Simple Model}

The previous observations prove useful in the practical implementation of a jump-to-default intensity model with a Markovian structure, as described in Bielecki et al. [5] (see also Ayache et al. [2] or Andersen and Buffum [1]). In [5], the filtration $\mathbb{F}$ is generated by a standard Brownian motion $W$ under $\mathbb{Q}$, and a primary market model composed of the savings account and $d=2$ risky assets is studied: 
- the first primary risky asset is the stock of a reference firm with price process $S$ and default time represented by $\tau_{d}$;

- the second primary risky asset is a credit default swap (CDS) written on the reference entity.

We choose as pre-default factor process $\mathcal{Z}$ the pair $(t, \widetilde{S})$, where $\widetilde{S}$ represents the pre-default stock price process, modeled as the unique strong solution to the following SDE

$$
d \widetilde{S}_{t}=\widetilde{S}_{t}\left(\left(r(t)-q(t)+\eta \gamma\left(t, \widetilde{S}_{t}\right)\right) d t+\sigma\left(t, \widetilde{S}_{t}\right) d W_{t}\right)
$$

with $\widetilde{S}_{0}$ given as a real-valued $\mathcal{F}_{0}$-measurable random variable, and where:

- the riskless short interest rate $r(t)$, the equity dividend yield $q(t)$, and the local default intensity $\gamma(t, S) \geq 0$ are bounded Borel functions,

- the fractional recovery on $S$ upon default, $\eta$, is a non-negative constant,

- the local volatility $\sigma(t, S)$ is a positively bounded Borel function,

- the functions $\gamma(t, S) S$ and $\sigma(t, S) S$ are Lipschitz in $S$.

It is further postulated that (cf. Subsection 4.1):

- the coupon process

$$
C_{t}=C(t):=\int_{[0, t]} c_{u}^{c b} d u+\sum_{0 \leq T_{i} \leq t} c^{i}
$$

for a bounded Borel continuous coupon rate $c^{c b}$ and deterministic discrete coupon dates and amounts, with $T_{0}=0$ and $T_{K-1}<T \leq T_{K}$;

- the recovery process $\bar{R}_{t}$ is of the form $\bar{R}\left(t, S_{t-}\right)$ for a Borel function $\bar{R}$.

We say that we deal with a hard call protection if the lifting time of call protection $\bar{\tau}=\bar{T}$ for some $\bar{T} \leq T$. A standard soft call protection corresponds to the lifting time of call protection given as $\bar{\tau}=\inf \left\{t>0 ; \widetilde{S}_{t} \geq \bar{S}\right\} \wedge T$ for some $\bar{S} \in \mathbb{R}_{+}^{*}$.

Proposition 4.2 Let us assume either a hard call protection or a standard soft call protection.

(i) Choosing the driver $f=f^{c b}$ as defined in (44), the related BSDE (E) is Markovian with respect to the factor process $\mathcal{Z}=(t, \widetilde{S})$ (so $\mathcal{X}$ is reduced to $\mathcal{X}^{1}=\widetilde{S}$ and there are no regimes; thus $d=$ $2, p=1, l=0)$.

(ii) The pre-default primary price process $\widetilde{S}$ satisfies the arbitrage drift condition (26).

(iii) In case $\bar{\tau}=0$ (no call protection), (34) becomes:

$$
\begin{gathered}
\max \left(\min \left(-\mathcal{L} \Theta+\mu \Theta-\left(\gamma R^{c b}+c^{c b}+a-\mu A\right), \Theta-\bar{P} \vee \kappa S\right), \Theta-\left(U^{c b}-A\right)\right)=0, \quad t<T, \\
\Theta(T, S)=\bar{N} \vee \kappa S,
\end{gathered}
$$

with $\mathcal{L} \equiv \partial_{t}+(r-q+\eta \gamma) S \partial_{S}+\frac{\sigma^{2} S^{2}}{2} \partial_{S^{2}}^{2}$ and $a_{t}=\frac{c^{i_{t}}}{T_{i_{t}}-T_{i_{t}-1}}$ (cf. Subsection 4.2). The function $\Theta$ defined by (46) is the clean pricing function of the related reduced convertible bond

Proof. (i) holds by construction of the model. Moreover, (the Markovian form of) condition (26) holds for $\widetilde{S}$ since in the present case

$$
\mu(t, S)=r(t)+\gamma(t, S), c(t, S)=q(t) S, \mathcal{R}(t, S)=(1-\eta) S,
$$

so that

$$
\mu(t, S) S-c(t, S)-\gamma(t, S) \mathcal{R}(t, S)=(r(t)-q(t)+\eta \gamma(t, S)) S
$$

which is the drift coefficient function of process $\widetilde{S}$. Thus (ii) is satisfied. Finally (iii) follows by specification of the general pricing equation (34) to the data of Proposition 4.1(i). 


\subsection{Comments on the Choice of the Driver in $(\mathcal{E})$}

We will now demonstrate, using the convertible bond example, the importance of the freedom to use the most convenient driver in $(\mathcal{E})$. This will illustrate and support the general comments on this issue made in Subsection 2.2 .

In Propositions 4.1 and 4.2 , the choice of the driver is motivated by the double objective that: - the jump times (if any) of $\mathcal{L}$ and $\mathcal{U}$ in the resulting doubly reflected $\operatorname{BSDE}(\mathcal{E}$ ) should be totally inaccessible (this excludes, in particular, jumps at deterministic discrete coupon times $T_{i}$ );

- $(\mathcal{E})$ should be a Markovian BSDE for a Markov factor process $\mathcal{Z}$ as simple as possible (with embedded process $\mathcal{X}$ having the dimension as low as possible, in particular).

The first requirement stems from the fact that general existence results for doubly reflected BSDEs typically postulate that $\mathcal{L}$ and $\mathcal{U}$ are quasi-left continuous processes; this is tantamount to the existence of sequence of totally inaccessible stopping times which exhaust their jump times (see [10, 16]).

When $\widetilde{S}$ is continuous, then under the choice 43 for $F$, this first requirement is met for $\mathcal{L}$, and, in case with no call notice period, for $\mathcal{U}$ as well, as Proposition 4.1(iii) shows $(\mathcal{L}$ and $\mathcal{U}$ are then simply continuous). It is further established in [5] that in the simple jump-diffusion model of Section 4.3 the process $\mathcal{U}$ is also continuous in the case of a convertible bond with positive call notice period.

The second requirement is, of course, motivated by the practical necessity to deal with a Markovian BSDE with a Markov factor vector process $\mathcal{Z}$ as low-dimensional as possible, for computational cost issues. As shown in Propositions 4.1 and 4.2 , the choice 43 for $F$ results in a model which is Markovian in $\widetilde{S}$ alone, in the simple jump-diffusion model of Section 4.3 .

Note, for instance, that by choosing $F=0$ instead of $F=\bar{F}+A$ in $(43)$, we would get

$$
\mathcal{L}=L^{c b}+\bar{F}=\bar{P} \vee \kappa \widetilde{S}+A+\alpha^{-1} \int_{[0, \cdot]} \alpha_{u} d \bar{D}_{u}
$$

where $A$ (resp. $\bar{F}$ ) jumps by $-c^{i}$ (resp. $c^{i}$ ) at time $T_{i}$. So, choosing $F=0$, the first requirement would be met for $\mathcal{L}$ (provided $\widetilde{S}$ is continuous), and, likewise (at least, in case with no call notice period), for $\mathcal{U}$. But the resulting doubly reflected $\operatorname{BSDE}(\mathcal{E})$ would not be Markovian in the simple jump-diffusion model of Subsection 4.3 at least not in $\widetilde{S}$ alone, because of the path dependence induced by the credit-risk adjusted discount factor $\alpha_{t}=\int_{0}^{t} \mu_{u} d u$ with $\mu_{t}=r(t)+\gamma\left(t, \widetilde{S}_{t}\right)$.

\section{A Appendix}

In this Appendix, we provide proofs of two results stated in the main body of the paper.

\section{A.1 Proof of Theorem 2.1}

Note that the first line of $(\mathcal{E})$ may be rewritten as

$$
\alpha_{t} \Theta_{t}=\alpha_{T} \chi+\alpha_{T} F_{T}-\alpha_{t} F_{t}+\int_{t}^{T} \alpha_{u} d K_{u}-\int_{t}^{T} \alpha_{u} d M_{u}, t \in[0, T]
$$

This is actually the way equation $(\mathcal{E})$ was presented in 4 , where a solution to $(\mathcal{E})$ was defined therein as a triplet $(\Theta, M, K)$ such that:

- $\Theta$ is a real-valued, $\mathbb{F}$-adapted, càdlàg process,

- $\int_{0}^{*} \alpha d M$ is a real-valued $\mathbb{F}$-martingale vanishing at time 0 ,

- $K$ is an $\mathbb{F}$-adapted finite variation process vanishing at time 0 ,

- all conditions in $(\mathcal{E})$ are satisfied, where in the third line $K^{+}$and $K^{-}$denote the Jordan components 
of $K$ (i.e., the decomposition $K=K^{+}-K^{-}$where the non-decreasing processes $K^{ \pm}$vanish at time 0 and define mutually singular measures), and where the convention that $0 \times \pm \infty=0$ is made in the third line.

Since $\alpha$ is a continuous and bounded process, it is thus rather immediate that a solution to $(\mathcal{E})$ in the sense of Definition 2.5 is also a solution to $(\mathcal{E})$ in the sense of [4, whereas the converse is true in the case where $\alpha$ is positively bounded, $M$ lies in $\mathcal{H}^{2}$ and $K$ is continuous.

Theorem 2.1. subsequently follows by application of Corollary C.1 in [4].

Remarks A.1 (i) The definition of a solution to $(\mathcal{E})$ as of 4 is better from the point of view of getting the most general results under minimal assumptions (see [4]). However the (more restrictive) Definition 2.5 is more convenient for practical purposes, like proving that the equation admits a unique solution, etc.

(ii) In [4] we defined more general notions of $\varepsilon$-hedges, that were pertaining in the case where there may be jumps in the process $K$. Since in all existing works on doubly reflected BSDEs the process $K$ is actually found to be a continuous process (see e.g. [10, 16, 5, 11]), we impose in this paper the continuity of $K$ in Definition 2.5, and we only consider hedges, not $\varepsilon$-hedges. Note however that Theorem 2.1 can be extended to possible jumps in $K$, using the generalized notion of $\varepsilon$-hedge defined in 4, and with the minimality conditions

$$
\int_{0}^{T}\left(\Theta_{u-}-\mathcal{L}_{u-}\right) d K_{u}^{+}=\int_{0}^{T}\left(\overline{\mathcal{U}}_{u-}-\Theta_{u-}\right) d K_{u}^{-}=0
$$

instead of

$$
\int_{0}^{T}\left(\Theta_{u}-\mathcal{L}_{u}\right) d K_{u}^{+}=\int_{0}^{T}\left(\overline{\mathcal{U}}_{u}-\Theta_{u}\right) d K_{u}^{-}=0
$$

in Definition 2.5. In the case where $K$ is continuous, both set of minimality conditions are equivalent, since the related integrands differ on an at most countable set and the integrators define atomless measures on $[0, T]$; see, e.g., [10].

\section{A.2 Proof of Lemma 3.1}

Let us introduce the Doléans-Dade martingale (see, e.g., [6])

$$
\mathcal{E}_{t}=\mathbb{1}_{\left\{t<\tau_{d}\right\}} e^{\Gamma_{t}}=1-\int_{0}^{t} \mathcal{E}_{u-} d N_{u}^{d}
$$

so that $\alpha_{t} \mathcal{E}_{t}=\beta_{t} \mathbb{1}_{\left\{t<\tau_{d}\right\}}$ and $\alpha_{t} \mathcal{E}_{t-}=\beta_{t} \mathbb{1}_{\left\{t \leq \tau_{d}\right\}}$. Then (cf. 24$\}$ )

$$
d\left(\beta_{t} \widehat{X}_{t}\right)=d\left(\mathcal{E}_{t} \alpha_{t} \widetilde{X}_{t}\right)+\beta_{t} d \mathcal{D}_{t}
$$

It may happen that $\alpha \widetilde{X}$ is not a $\mathbb{G}$-semimartingale, so a direct application of the (G-)integration by parts formula to $\mathcal{E} \alpha \widetilde{X}$ is precluded. However, $\alpha \widetilde{X}$ stopped at $\tau_{d}$ is a $\mathbb{G}$-semimartingale, and since the process $\mathcal{E}$ is killed at $\tau_{d}$, we have that $\mathcal{E} \alpha \widetilde{X}=\mathcal{E} \alpha{ }_{\cdot \wedge \tau_{d}} \widetilde{X}_{\cdot \wedge \tau_{d}}$. Hence, by an application of the integration by parts formula to $\mathcal{E}_{{ }_{\cdot \wedge \tau_{d}}} \widetilde{X}_{\cdot \wedge \tau_{d}}$, we obtain

$$
d\left(\mathcal{E}_{t} \alpha_{t} \widetilde{X}_{t}\right)=d\left(\mathcal{E}_{t} \alpha_{t \wedge \tau_{d}} \widetilde{X}_{t \wedge \tau_{d}}\right)=\mathcal{E}_{t-}\left(d\left(\alpha_{t} \widetilde{X}_{t}\right)-\alpha_{t} \widetilde{X}_{t-} d N_{t}^{d}\right)+d\left[\mathcal{E}, \alpha_{\cdot \wedge \tau_{d}} \widetilde{X}_{\cdot \wedge \tau_{d}}\right]_{t},
$$

where, in addition, we have that $\left[\mathcal{E}, \alpha \cdot \wedge \tau_{d} \widetilde{X}_{\cdot \wedge \tau_{d}}\right]_{t}=-e^{\Gamma_{\tau_{d}}} \alpha_{\tau_{d}} \Delta \widetilde{X}_{\tau_{d}} H_{t}$. Now, in view of our standing assumption that $\tau_{d}$ avoids $\mathbb{F}$ - stopping time, and since $\widetilde{X}$ is a càdlàg process, therefore $\Delta \widetilde{X}_{\tau_{d}}=0$. Using (23), we then deduce from (47) that

$$
\begin{aligned}
& d\left(\beta_{t} \widehat{X}_{t}\right)=\mathcal{E}_{t-}\left(d\left(\alpha_{t} \widetilde{X}_{t}\right)-\alpha_{t} \widetilde{X}_{t-} d N_{t}^{d}\right)+\beta_{t} d \mathcal{D}_{t} \\
& \quad=\mathbb{1}_{\left\{t \leq \tau_{d}\right\}} \beta_{t}\left(\alpha_{t}^{-1} d\left(\alpha_{t} \bar{X}_{t}\right)-d \overline{\mathcal{D}}_{t}-\widetilde{X}_{t-} d N_{t}^{d}\right)+\beta_{t} d \mathcal{D}_{t} \\
& =\mathbb{1}_{\left\{t \leq \tau_{d}\right\}} \beta_{t}\left(\alpha_{t}^{-1} d\left(\alpha_{t} \bar{X}_{t}\right)+\left(\mathcal{R}_{t}-\widetilde{X}_{t-}\right) d N_{t}^{d}\right)
\end{aligned}
$$


where the last equality uses the fact that the $\mathbb{F}$ - (hence $\mathbb{G}$-) predictable coupon process $\mathcal{C}$ cannot jump at the $\mathbb{G}$-totally inaccessible stopping time $\tau_{d}$.

\section{References}

[1] Andersen, L. and Buffum, L.: Calibration and implementation of convertible bond models. Journal of Computational Finance 7 (2004), 1-34.

[2] Ayache, E., Forsyth, P. and Vetzal, K.: Valuation of convertible bonds with credit risk. The Journal of Derivatives, Fall 2003.

[3] Bielecki, T.R., Crépey, S., Jeanblanc, M. and Rutkowski, M.: Arbitrage pricing of defaultable game options with applications to convertible bonds. Forthcoming in Quantitative Finance.

[4] Bielecki, T.R., Crépey, S., Jeanblanc, M. and Rutkowski, M.: Valuation and hedging of defaultable game options in a hazard process model. Working Paper available online at www.defaultrisk. com, 2007.

[5] Bielecki, T.R., Crépey, S., Jeanblanc, M. and Rutkowski, M.: Convertible Bonds in a Defaultable Diffusion Model. Working Paper available online at www.defaultrisk.com, 2007.

[6] Bielecki, T.R. And Rutkowski, M.: Credit Risk: Modeling, Valuation and Hedging. Springer-Verlag, Berlin Heidelberg New York, 2002.

[7] Cherny, A. And Shiryaev, A.: Vector stochastic integrals and the fundamental theorems of asset pricing. Proceedings of the Steklov Institute of Mathematics 237 (2002), 6-49.

[8] Crépey, S.: About the Pricing Equation in Finance. Submitted.

[9] Crépey, S., Matoussi, A.: About the Greeking Equation in Finance. Work in preparation.

[10] Crépey, S., Matoussi, A.: Reflected and Doubly reflected BSDEs with jumps: A priori estimates and comparison principle. Submitted.

[11] Cvitanić, J. and Karatzas, I.: Backward stochastic differential equations with reflection and Dynkin games. Annals of Probability 24 (1996), 2024-2056.

[12] Delbaen, F. and Schachermayer, W.: The fundamental theorem of asset pricing for unbounded stochastic processes. Mathematische Annalen 312 (1997), 215-250.

[13] Dellacherie, C., Maisonneuve, B. and Meyer, P.-A.: Probabilités et Potentiel, Chapitres XVII-XXIV. Hermann, Paris, 1992.

[14] El Karoui, N., Peng, S., And Quenez, M.-C.: Backward stochastic differential equations in finance. Mathematical Finance 7 (1997), 1-71.

[15] Ethier H.J. And Kurtz, T.G.: Markov Processes. Characterization and Convergence. Wiley, 1986.

[16] Hamadène, S. And Hassani, M.: BSDEs with two reflecting barriers driven by a Brownian motion and an independent Poisson noise and related Dynkin game. Electronic Journal of Probability 11 (2006), 121-145.

[17] JACOD, J.: Calcul Stochastique et Problèmes de Martingales. Springer, Berlin Heidelberg New York, 2003.

[18] Jacod, J. And Shiryaev, A. N.: Limit Theorems for Stochastic Processes. Springer, Berlin Heidelberg New York, 1979. 
[19] Kallsen, J. And KüHn, C.: Convertible bonds: financial derivatives of game type. In: Exotic Option Pricing and Advanced Lévy Models, Edited by Kyprianou, A., Schoutens, W. and Wilmott, P., Wiley, 2005, pp. 277-288.

[20] KifER, Y.: Game options. Finance and Stochastics 4 (2000), 443-463

[21] MA, J. And Yong, J.: Forward-backward stochastic differential equations and their applications. Lecture Notes in Mathematics 1702, Springer, Berlin Heidelberg New York, 1999.

[22] Mansuy, R. And Yor, M.: Random times and enlargement of filtrations in a Brownian setting. Lecture Notes in Mathematics 1873, Springer, Berlin Heidelberg New York, 2005.

[23] A. Nikeghbali and M. Yor: A definition and some characteristic properties of pseudostopping times. Ann. Probab. 33 (2005), 1804-1824.

[24] Protter, P.E.: Stochastic Integration and Differential Equations. Second edition, Springer, Berlin Heidelberg New York, 2004. 